\title{
3D Quantitative Shape Analysis on Form, Roundness, and Compactness with $\mu \mathrm{CT}$
}

\author{
Budi Zhao ${ }^{1}$ and ${ }^{*}$ Jianfeng Wang ${ }^{1}$ \\ ${ }^{1}$ Department of Architecture and Civil Engineering, City University of Hong Kong, Hong Kong, China
}

\begin{abstract}
Particle shape plays an important role in determining the engineering behaviour of granular materials. In this regard, characterisation and quantification of particle shape are essential for understanding the behaviour of granular materials. X-ray micro-computed tomography $(\mu \mathrm{CT})$ enables observation of particle morphology at ever-greater resolutions. The challenge has thus become extracting quantified shape parameters from these rich three-dimensional (3D) images. In this paper, we implement X-ray $\mu \mathrm{CT}$ to obtain $3 \mathrm{D}$ particle morphology and utilize image processing and analysis techniques to quantify it at different scales. A novel framework is proposed to measure 3D shape parameters of form, roundness, and compactness. New 3D roundness indexes were formulated from the local curvature on reconstructed triangular surface mesh. Subsequently, this method is utilized to study the change of particle shape by single particle crushing tests on Leighton Buzzard sand (LBS) particles. It is found that compactness value (i.e., sphericity) could be influenced by both form and roundness. Then, the distributions of shape parameters are characterized by Weibull statistics. It shows that single particle crushing tests generates more irregular fragments which have smaller shape parameters with larger variance for the measured shape parameters.
\end{abstract}

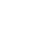
)

Keywords: Compactness; Form; Reconstructed surface mesh; Roundness; Voxel assembly; X-ray tomography.

\footnotetext{
*Corresponding author. Tel.: +852-3442-6787. E-mail: jefwang@ cityu.edu.hk.
} 


\section{$1 \quad 1$ Introduction}

2 The analysis of particle shape and its influence on the mechanical behaviour of granular ma3 terials has been the subject in geology and engineering science for a long time. Particle shape 4 influences attainable density, stiffness, compressibility and critical state parameters, such as 5 critical state friction angle [e.g., 1-3]. The end resistance of cone penetration test (CPT) at 6 a given relative density and stress level is affected significantly by particle shape [4]. On the 7 other hand, particle damage under elevated stress levels changes particle shape, e.g. smaller 8 particles which are less spherical, less convex and with lower aspect ratio are generated when 9 particles suffer catastrophic splitting [5]. Numerical modelling, e.g. discrete element method 10 and molecular dynamics method, has been shown to constitute an important way to understand 11 the influences of particle shape on the behaviour of granular materials [e.g., 6-10]. Although 12 comprehensive laboratory and numerical tests have been conducted, there are few techniques 13 to predict the mechanical behaviour of irregularly shaped particles. The lack of models and 14 correlations can be attributed to the difficulty of defining and measuring shape descriptors for

15 16 17 18 19 a wide variety of complex particle shapes.

Most of widely implemented rules to quantify particle shape have been developed by sedimentary geologists, which are qualitative shape determinations made by two-dimensional (2D) visual classification. These methods are time-consuming and too subjective to generate parameters that could be utilized for simulating and modelling for geotechnical engineering purposes. Acknowledging these drawbacks, many researchers adopted advanced devices (e.g., optical microscopy, laser beam systems, interferometer, and X-ray $\mu \mathrm{CT}$ ) and robust numerical methods to obtain more comprehensive quantitative particle shape indexes. These shape quantification methods can be divided into two groups, i.e. two-dimensional (2D) methods and three-dimensional (3D) methods.

Two-dimensional methods collect 2D projections from scanning electron microscope (SEM), optical microscopy or laser beam systems and implement digital image processing techniques to quantify particle shape. Various approaches have been proposed for characterizing particle shape from the collected 2D projections. Generally, common 2D shape factors indicate the aspect ratio of two dimensions (e.g., Feret dimensions and principal dimensions) or describe the deviations of the shape of a particle from the circular shapes (e.g., the area-equivalent circle 

and the inscribed circle). This kind of evaluation technique has been combined with highspeed camera in many commercial 2D particle size and particle shape analysis systems, e.g., DiaInspect.OSM (Vollstädt-Diamant GmbH), CAMSIZER (Retsch Technology) and QICPIC (Sympatec GmbH). Examples of these systems' application include obtaining the database of sand particles [11] and quality control of abrasive materials [12]. Theoretical model is also shown to have significance in examining the relationship between these shape factors [13]. Recently, a novel method has been proposed to quantify roundness, a scale dependent variable, by fitting circles to the identified corners of a particle outline [14]. A more complex and theoretical method, Fourier mathematical method, has also been developed. It was found that Fourier descriptors, which are determined by taking the Fourier transformation of the outline of sand particles, were closely related to shape factors $[15,16]$. Although 2D methods can characterize and quantify shape characteristics, noticeable difference between 2D and 3D shape indexes have been found $[17,18]$. Indexes based on 2D projects may highly depend on the choice of observing directions, which will result in non-unique shape descriptors for a given individual particle.

Three-dimensional particle morphology is usually measured by X-ray $\mu \mathrm{CT}$ or $3 \mathrm{D}$ laser scanner, and then processed to retrieve particle surface information. Image analysing methods are implemented to quantify particle shape from the surface information. In general, these methods could be classified into three groups. The first one has been performed on voxel assembly to obtain particle orientation, principal dimensions, volume and surface area [17-19]. This method usually leads to a large variance in estimated surface area due to the fact that different types of boundary voxels are not well distinguished and classified. This problem has been solved by identifying the local configuration of each boundary voxel using Marching Cubes algorithm, and assigning a surface area weight to each configuration [20]. The second method is based on reconstructed surface, which is composed of triangular surface meshes. It can be obtained by a three-dimensional laser scanner [21] or performing Marching Cube algorithm on voxel assembly [19]. The triangular mesh surface provides a more accurate representation of particle surface than the voxel assembly, which resulted in a more precise surface area estimation. A more complex and theoretical method performs 3D spherical harmonic analysis to mathematically characterize the morphology of particles, and then calculates shape factors [22-27]. However, it does not seem to provide an efficient way to obtain shape descriptors after 


\section{Methodology}

$11 \mathrm{X}$-ray $\mu \mathrm{CT}$ and 3D laser scanner are two common instruments that can be used to acquire 3D 12 surface information for particle shape analysis. Due to the limited resolution, 3D laser scanner 13 is not suitable for small sand particles, e.g. $1 \mathrm{~mm}-2 \mathrm{~mm}$ LBS particles measured in this study. 14 Therefore, we implemented X-ray $\mu \mathrm{CT}$ scanning to obtain $3 \mathrm{D}$ gray-level CT images. These 15 images were put through a series of image processing and analysis methods to obtain different $163 \mathrm{D}$ particle morphology data and shape factors, respectively. This procedure is shown in Fig. 1, 17 and will be described in detail in the following parts. The gray-level CT images were first pro18 cessed to generate the voxel assembly, which represents sand particles with a group of discrete 19 voxels. Then, surface reconstruction methods were implemented to extract a smooth isosurface, 20 representing more precise particle surface with triangular meshes. The reconstructed surface 21 was simplified to remove surface texture for evaluating the scale-dependent particle roundness. 22 In summary, three types of data were obtained after image processing, i.e., voxel assembly, 23 smooth triangular surface mesh and simplified triangular surface mesh. Then, image analysing 24 methods were applied to these data to quantify particle shape at different scales, e.g. form and 25 roundness. 


\subsection{X-ray $\mu \mathrm{CT}$ image acquisition and processing}

There has been a very rapid development of X-ray instrumentation, mainly including laboratory $\mu \mathrm{CT}$ systems and synchrotron $\mu \mathrm{CT}$, as reviewed by Stock [28]. Commercial lab $\mu \mathrm{CT}$ systems designed for studying specimens with diameters about $10 \mathrm{~mm}$ can be expected to produce highest resolutions with voxels between 1 and $10 \mu \mathrm{m}$ in size. X-ray $\mu \mathrm{CT}$ scan collects a series of projections during the rotation of an object between an X-ray source and a detector. The data collection rate can vary widely for a single instrument depending on chosen operating conditions. These projections could be reconstructed to a 3D image with the gray value reflecting the material density. Reconstruction time depends on variables such as computer architecture and number of processors. The typical time used for data collection and reconstruction of one sample in this study is about 1.5 hours and 10 minutes, respectively. Synchrotron $\mu \mathrm{CT}$ has a significant advantage over lab $\mu \mathrm{CT}$ systems that it could generate higher intensity beams to obtain an image of higher quality with less noise and higher contrast between different materials. However, the commercial lab $\mu \mathrm{CT}$ systems have much easier accessibility than synchrotron $\mu \mathrm{CT}$ sources.

In this study, LBS particles were scanned with the nanome|x system (Phoenix, General Electric Company (GE)) located at the Advanced Engineering Materials Facilities (AEMF) of the Hong Kong University of Science and Technology. To increase the scanning efficiency, a plastic container was specially designed for scanning 28 particles at a time, at the cost of reducing scanning resolution. The container has seven holes of $2.5 \mathrm{~mm}$ diameter. Twenty-eight particles were put into these holes separately. This X-ray machine has a vertically placed X-ray tube and a horizontal placed rotational axis. Therefore, silicone grease was used to attach these particles to the container to prevent them from moving around during rotation. The satisfactory reconstructed image shows that silicone grease provides sufficient support for particles with current weight. The obtained projections from these scanning systems were reconstructed to 3D gray images using Phoenix Datos|x CT, a software using the Feldkamp filtered back projection algorithm. The LBS particles were scanned at $120 \mathrm{KeV}$ energy with a spatial resolution of about $15 \mu \mathrm{m} /$ voxel. The data of LBS fragments generated from in-situ single particle crushing tests were obtained in our previous study which has been described by Zhao et al. [29]. The v|tome|x m (Phoenix, GE), located at Shanghai Yinghua NDT Equipment Trade Co., Ltd., was 
utilized to obtain $\mu \mathrm{CT}$ images with a resolution of about $3 \mu \mathrm{m}$.

The reconstructed 3D gray images were put through several image processing techniques to reduce image noise and to segment different phases. An example of the results of image processing on a LBS particle is shown in Fig. 2. A 3D median filter was applied to reduce noise typically found in raw CT data, which replaces the intensity value of a voxel by the median of the intensity values in its neighbourhood [30]. The implemented filter size is $3 \times 3 \times 3$, and it is repeated three times. Fig. 2(b) shows that the filter efficiently smoothed the image without a significant influence on the outline of the particle. Thresholding segmentation was utilized to separate the filtered images into different regions based on intensity level. The intrinsic function, multithresh, from MATLAB (Mathworks, Natick, MA, USA) was implemented to obtain the threshold values based on the multilevel Otsu's threshold method [31]. Thresholding segmentation separates the sand particles from other materials (Fig. 2(c)). Fig. 2(d) shows that the particles are successfully separated from voids, silicone grease and the plastic container with seven holes. The resultant images are 3D voxel assemblies composed by 1 and 0 , which represent particle and other materials, respectively.

\subsection{Reconstruction of 3D particle surface}

Surface reconstruction is an important step in geometric modeling by extracting a polygonal mesh of an isosurface from 3D scalar voxels. It allows one or more phases of interest in data set to be isolated and rendered. The Marching Cubes (MC) method proposed by Lorensen and Cline [32] is probably the most popular isosurface algorithm. It has been widely applied in many areas, including biochemistry [33], biomedicine [34], and deformable modelling [35], etc. Figs. 3 (a) and (d) show the voxel assembly of a typical LBS particle, which consists of about 1.3 million voxels, and its close-up view. The MC algorithm produces a high-resolution 3D surface well representing the overall particle shape (Fig. 3 (b)). However, the surface generated from MC have artificial stair-steps which makes a highly rough surface (Fig. 3 (e)). Therefore, smoothing is necessary to remove the jagged stair-steps and at the same time preserve the overall shape. A variety of algorithms have been proposed to reconstruct the smooth surface in recent years, e.g., Laplacian iterative smoothing [36] and feature-sensitive surface extraction [37]. In this study, particle surface was reconstructed using a generalised Marching 
1 Cubes algorithm (GMC) with a Gaussian filter [38, 39]. This method assigns probabilities to 2 the vertices and interpolates them. The probability information was edited through a modified 3 Gaussian filter with a given kernel size. Figs. 3 (c) and (f) show that GMC could efficiently 4 reduce the stair-steps with a kernel size of 5 voxels.

\subsection{D particle shape quantification at different scales}

6 There are three important scales in particle shape, i.e., form (overall shape), roundness (or an7 gularity), and roughness. As illustrated in Fig. 4, form reflects the proportions of a particle; 8 roundness reflects variations at corners, i.e., variations superimposed on form; and roughness 9 reflects the surface texture imposed on corners and between corners [40]. They are essen10 tially independent because one can vary widely without necessarily affecting the other two 11 properties. For example, change in surface texture needs not affect roundness, e.g., weather12 ing may increase surface roughness while preserve the well-rounded corners. Roundness of 13 rock particles, which normally increases through abrasion, can change greatly without much

14 effect on form. Roughness, which describes the smallest shape scale, is usually studied in an 15 area of about $20 \times 20 \mu \mathrm{m}^{2}$ with interferometer which could provide a resolution around $1 \mathrm{~nm}$ $16[5,18,41,42]$. It seems impractical to characterize particle roughness at the current X-ray $\mu \mathrm{CT}$ 17 resolution. In this case, we only focus on particle form and roundness.

\section{$18 \quad$ 2.3.1 Particle form}

19 As summarised by Barrett [40] and Blott and Pye [43], almost all parameters of particle form 20 are evaluated by the three principal dimensions at the long axis $(a)$, intermediate axis $(b)$, and 21 short axis (c), where, $a>b>c$. Zingg [44] plotted two ratios, $b / a$ and $c / b$, on a bivariate dia22 gram and used the ratio values of $2 / 3$ to define four shape classes, i.e., spheroid, oblate, prolate, 23 and blade. Although form is essentially a 3D concept, many researchers made the 2D estima24 tion based on Feret's diameters or visual comparison charts, because of their relative simplicity. 25 An apparent disadvantage of all 2D estimates of form is that they cannot differentiate between 26 discs and spheres of similar outlines.

27 The orientations of the major, minor, and intermediate axes for each particle could be deter28 mined by principal component analysis (PCA) on the vertexes of reconstructed surface. This 
method has been applied to the analysis of tomographic data in different fields $[17,45]$. The particles were then rotated so that their principal axes of inertia are parallel to the Cartesian axes. The three principal dimensions, i.e., the major dimension $(a)$, the intermediate dimension (b) and the minor dimension $(c)$, were then determined by measuring the coordination dimensions. Based on the principal dimensions, two aspect ratios, i.e., the elongation index (EI) and the flatness index $(\mathrm{FI})$, were defined as: $\mathrm{EI}=b / a$ and $\mathrm{FI}=c / b$.

\subsubsection{Particle roundness}

Most methods for measuring roundness depend on the curvature of the corners on particle surface [43]. However, the existing 3D roundness indexes usually do not involve corner identification and curvature evaluation $[18,46]$. In this study, we propose a novel method to evaluate $3 \mathrm{D}$ roundness based on local curvature values. The procedure to obtain 3D roundness indexes in three steps is explained as follows, i.e. mesh simplification for the cut-off between roundness and roughness, local curvature estimation, and calculation of particle roundness indexes. Then, we illustrate the influence of mesh simplification on the local curvature estimation.

\section{1) Mesh simplification for the cut-off between roundness and roughness}

Particle roughness and remaining stair-steps on reconstructed surface should be removed before roundness evaluation. Previous researchers removed roughness by dicretising the outline of a particle into an $\mathrm{N}$-side equal angle polygon [e.g., 47]. The selected $\mathrm{N}$-value could therefore serve as the cut-off between angularity and roughness. Sukumaran and Ashmawy [47] suggested that $\mathrm{N}=40$ would be appropriate for $2 \mathrm{D}$ condition. When it is extended to $3 \mathrm{D}$ condition, it is equivalent to about 1,200 triangular meshes. In this study, we adopt this approach and reduce the number of triangular surface meshes by mesh simplification. An edge-collapsing algorithm which is controlled by setting a minimum number of surface elements is implemented [48], and the number of mesh for each particle is fixed as 1,500. The effects of this simplification on reconstructed surface will be examined later.

\section{2) Local curvature estimation}

A brief explanation of 3D surface curvature is described as follows. For a 3D regular surface $(\boldsymbol{S})$, a point $\boldsymbol{p}$ lies on it with the unit length normal $\boldsymbol{N}$ (Fig. 5[a]). Given a unit vector $\boldsymbol{T}$ in the tangent plane to $S$ at $\boldsymbol{p}$, we can pass through $\boldsymbol{p}$ a curve $\boldsymbol{C} \subset \boldsymbol{S}$, which has $\boldsymbol{T}$ as its tangent vector at $\boldsymbol{p}$. According to Do Carmo [49], all curves lying on $\boldsymbol{S}$ and having the same tangent vector $\boldsymbol{T}$ at $\boldsymbol{p}$ 
have the same normal curvature at this point. Among all of these curves, a particular one is the normal section of $\boldsymbol{S}$ at $\boldsymbol{p}$ along $\boldsymbol{T}$, which is obtained by intersecting $\boldsymbol{S}$ with a plane containing $\boldsymbol{T}$ and $\boldsymbol{N}$. If we let the unit vector $\boldsymbol{T}$ rotate around $\boldsymbol{N}$, we can define an infinite number of normal sections, each of which is associated with a normal curvature. Among them, there are two sections, which occur in orthogonal directions, whose normal curvature attains maximum and minimum, respectively. These two normal curvatures are the maximum principal curvature $\left(k_{1}\right)$ and the minimum principal curvature $\left(k_{2}\right)$. Based on the principal curvatures, the mean curvature $\left(k_{M}\right)$ and the Gaussian curvature $\left(k_{G}\right)$ are defined, respectively, as:

$$
k_{M}=\left(k_{1}+k_{2}\right) / 2
$$

and

$$
k_{G}=k_{1} k_{2}
$$

The 3D local curvatures on triangular surface mesh could be estimated using the method described by Chen and Schmitt [50] and Dong and Wang [51]. The triangular mesh on particle surface was looked as an approximation of a smooth surface. The goal of this algorithm is to estimate the principal directions and principal curvatures at each vertex on the surface mesh. This task is fulfilled through estimating the normal vectors at each vertex, approximating the normal curvature, selecting the maximum normal curvature, and estimating the minimum principal curvature and principal directions. The principal curvatures at a vertex could be estimated from a one-ring neighborhood or higher-ring neighborhood, as shown in Fig. 5(b). The impact of noise and mesh irregularity could be reduced by a higher-ring neighborhood. To simplify this study, we used the algorithm with a one-ring neighborhood. This algorithm was described in detail by Dong and Wang [51].

\section{3) Definition of particle roundness indexes}

The local curvature values evaluated at each vertex indicate how much a local surface deviates from a flat plane, e.g. a smaller curvature value represents a flatter surface. However, curvature values need to be normalized by particle size before being used to quantify particle shape, e.g., spheres with different sizes have different local curvatures with respect to their radii. Therefore, we first define the local roundness at each vertex on particle surface as $k_{s} / k_{i}$, where $k_{i}$ is one kind of the local curvature at this vertex, which could be $k_{1}, k_{2}, k_{M}$, and $k_{G}$, and $k_{s}$ is the corresponding curvature of the particle's maximum inscribed sphere. The maximum 
inscribed sphere could be determined using a 3D distance map. For each voxel inside the sand particle, the distance to the nearest boundary voxel is computed, resulting in the distance map as shown in Figs. 6 (a) (LBS particle) and (b) (LBS fragment). The largest distance value and its location identify the radius and the center of the maximum inscribed sphere. The similar method was utilized by Zheng and Hryciw [14] to obtain the maximum inscribed circle from 2D particle outlines, whereas 3D algorithm was used in this study. Figs. 6 (c) and (d) show the 3D view of the maximum inscribed sphere inside a LBS particle and a LBS fragment, respectively. The roundness of each triangular element is calculated as the average roundness of the three vertices that define the element. The triangular element having a roundness value smaller than one, meaning that the local curvature value is larger than its inscribed sphere's curvature value, is treated as a part of a "corner" and used to evaluate the particle's roundness.

Fig. 7 shows the "corners" identified by four different kinds of local curvatures on the reconstructed surface of a typical LBS particle with 1,500 faces. It turns out that the "corners" identified by different curvatures are distinct from each other. Maximum principal curvature determines "corners" cover almost all of the particle surface (Fig. 7 (a)), while minimum principal curvature and Gaussion curvature identify very few "corners" (Figs. 7 (b) and (d)). In this paper, we select mean curvature to identify corners and evaluate roundness because it provides well identified corners and has smooth distribution on the corners.

Finally, the 3D roundness indexes of a particle were defined as the area-based average roundness as:

$$
R_{i}=\frac{\sum\left(A_{n} \frac{k_{s}}{k_{i}}\right)}{\sum\left(A_{n}\right)}
$$

where $A_{n}$ is the area of the $n^{\text {th }}$ triangular element, which is part of the corners on the particle; and $R_{i}$ is the roundness value evaluated from different kinds of curvature values. The maximum value of roundness achieved by this formula is 1.00 for a particle that has all local curvatures smaller than, or equal to, the curvature of its maximum inscribed sphere.

\section{4) Illustration of mesh simplification on reconstructed surface}

In order to investigate the influence of mesh simplification, the reconstructed surfaces of a LBS particle and a LBS fragment were simplified with different number of faces, i.e. 10,000, 4,000, 1,500 and 500. Fig. 8 shows the 3D view of surfaces and local mean curvature values at the corners where the mean curvatures are larger than the curvature of its maximum inscribed sphere. It shows that the identified corners on the surfaces with 10,000 faces (Figs. 8 (a) and 
(e)) are influenced by the surface textures. As the number of faces decreasing, the influence of surface textures is decreasing or completed eliminated, and the corners are well identified with 1,500 faces for both LBS particle and fragment. Fig. 9 shows the influence of simplification on cumulative distributions of the mean curvature. It shows that simplification makes curvature distributions more and more concentrated to the curvature value of its inscribed sphere. The removing of surface textures contributes to the change of curvature distributions. However, if the number of faces continues to reduce, angularity features on the surface will also be removed, and the surface will be more and more close to a sphere. For example, the surface of LBS fragment with 200 faces has almost no faces with mean curvature larger than $7 \mathrm{~mm}^{-1}$. It shows that the investigation on identified corners and distributions of local curvature values is helpful for determining an appropriate number of faces for roundness evaluation. However, it remains an open problem to determine the most appropriate value.

\subsubsection{Particle compactness}

The concept of "sphericity" proposed by Wadell [52] is usually referred to as a global form of particles. It was defined as the ratio of the surface area of a sphere with the same volume as the given particle to the surface area of the particle.

$$
S=\sqrt[3]{36 \pi V^{2}} / S A
$$

where $V$ is the volume of the particle, and $S A$ is the surface area of the particle. In this study, particle volume and surface area are measured from the reconstructed surface. Particle volume is the volume bounded by reconstructed surface, and surface area is the total area of triangular elements. Surface area measurement is controversial because instruments with different resolutions and scaning length scales will yield different levels surface details. In general, the reconstructed surface provides the best estimate of surface area of the particle based on the current $\mu \mathrm{CT}$ resolution (Figs. 3 (e) and (f)). It has been noted that the surface area measured from voxel assembly is usually overestimated [19].

5 Sphericity is a concept that has been frequently confused with form [40, 43]. For example, 6 many researchers used sphericity to describe particle form, as summarised by Clayton et al. [53]. In fact, sphericity is an overall shape parameter that could be influenced independently 8 by form, roundness, and roughness. It is because the surface area of a particle could be signifi- 

of the particle $(V)$ and the volume of the convex hull $\left(V_{C H}\right)$ enclosing the particle:

$$
C_{X}=V / V_{C H}
$$

6 A convex hull is defined as the minimal convex surface containing all voxels of the component.

7 It is obtained through the intrinsic function "convhulln" in Matlab (Mathworks).

\section{Application to LBS Particles and LBS Fragments}

9 In this study, the above framework of morphology quantification was used to examine the 10 morphology change of Leighton Buzzard sand (LBS) made by single particle crushing tests. 11 LBS particles are mainly composed of quartz, which is chemically stable. Their shape and 12 surface textures are mainly modified by geological transportation processes. We randomly 13 chose 84 LBS particles with minimum dimensions between about $1 \mathrm{~mm}$ and $2 \mathrm{~mm}$. The LBS 14 fragments were obtained from the single particle crushing tests of four LBS particles. Fifty 15 fragments were randomly retrieved from the scans taken at the final loading stages of in-situ 16 tests. The details of the tests were described by Zhao et al. [29].

Fig. 10 shows 3D view of reconstructed surfaces of four LBS particles and four LBS 18 fragments with different sphericity values. LBS particles have regular form and smooth corners 19 due to geological transportation process, while LBS fragments have irregular form and sharp 20 corners generated by intersection of fracture planes. For LBS particles, particle roundness 21 changes more significantly than particle form as sphericity value reduces from 0.94 to 0.80 , 22 whereas particle form of LBS fragments changes more significantly than particle roundness as 23 sphericity value reduces from 0.82 to 0.50 .

\section{$24 \quad 3.1 \quad$ Results of quantitative shape analysis}

25 The aspect ratios of LBS particles and LBS fragments were plotted in Fig. 11. According to the 26 classification proposed by Zingg [44], most LBS particles could be classified into "spheroid" 
with both EI and FI larger than 2/3. The LBS fragments have lower values and a more disperse distribution of aspect ratios, which means that single particle crushing tests generated more irregular fragments in terms of particle form.

Fig. 12 shows the resultant relationship between $R_{M}$ and other roundness values of the tested particles. It appears that there is a strong correlation between these four roundness values, especially for LBS particles. $R_{2}$ is usually the largest roundness value, followed in decreasing order by $R_{M}, R_{G}$, and $R_{1}$. It appears that the roundness values exhibit a slight decrease from LBS particles to LBS fragments, with the average $R_{M}$ decreasing from 0.71 to 0.66 . A more scattered distribution is found for LBS fragments.

Sphericity is an important shape parameter to be considered for the characterization of 3D particle shape. Fig. 13 shows the log-log plot of surface area versus volume for the tested particles. The lines representing sphere particles and particles with sphericity equals to 0.8 and 0.5 were also included in Fig. 13. It shows that most LBS particles are between the lines of spheres and $\mathrm{S}=0.8$, while most LBS fragments are between the lines of $\mathrm{S}=0.5$ and $\mathrm{S}=0.8$.

Although sphericity and convexity both describe the compactness of particles, they described it from different aspects. Firstly, sphericity is a fraction of surface area while convexity is a fraction of volume. Secondly, sphericity compares the particle with its volume-equivalent sphere; whereas, the convexity compares that with its convex hull. Fig. 14(a) shows the relationship between measured sphericity and convexity values. Most LBS particles have sphericity and convexity values larger than 0.8 ; whereas, most LBS fragments have smaller sphericity and convexity values from 0.5 to 0.8 . It is found that a strong relationship exists between the sphericity and convexity values of both LBS particles and LBS fragments. To make further comparison between these two compactness measurements, sphericity was changed to a volume fraction through:

$$
S^{1.5}=6 V /\left(S A^{1.5} / \sqrt{\pi}\right)=V / V_{\text {sphere }}
$$

where $V_{\text {sphere }}$ is the volume of a sphere with the same surface area of the particle. Fig. 14(b) shows that most particles have larger $C_{X}$ than $\mathrm{S}^{1.5}$, which means that $V_{\text {sphere }}$ is larger than $V_{C H}$. This is obvious because of two reasons. Firstly, a sphere has the smallest ratio between its surface area and volume among all geometries, i.e., $S A / V_{\text {sphere }} \leq S A_{C H} / V_{C H}$. Secondly, the surface area of the sphere, which equals the surface area of the particle, is larger than or equal to that of its convex hull, $S A \geq S A_{C H}$. It is because that surface area is reduced by reducing the 
concave features of surface. Then, it is simple to obtain that $V_{s p h e r e} \geq V_{C H}$.

Although Wadell [52] stated that sphericity and roundness are two separate aspects of particle shape, they are actually not independent. As illustrated previously, sphericity is a shape parameter that is related to form, roundness and roughness. Fig. 15 (a) shows the contour map of the sphericity values of all measured particles. This graph clearly shows how the variations of form and roundness influence sphericity values. Generally, the particles that have larger sphericity values also have larger values of representative aspect ratio (AR) and mean roundness value $\left(R_{M}\right)$. AR is defined as the average value of EI and FI. For irregular particles (mostly LBS fragments) with sphericity values smaller than 0.8 , particle form dominates the change of sphericity values; whereas, for regular particles (mostly LBS particles) with sphericity values larger than 0.8 , roundness shows more significant influence on the change of sphericity values. Two LBS particles (A-B) and four LBS fragments (C-F) were identified from Fig. 15 to visually illustrate the shape parameters. Fig. 16 shows the 3D view of reconstructed surface with mean curvature distribution on corners along with quantitative shape parameters. Particle $\mathrm{A}$, which is close to a cube, has a very high $\mathrm{AR}$ value and relatively low $R_{M}$. Particle $\mathrm{B}$ has a lower AR value than particle $\mathrm{A}$, but it has higher values of $R_{M}$ and S. It shows a more significant influence of roundness on the sphericity values than form for LBS particles. For LBS fragments, $R_{M}$ decreases from $\mathrm{C}$ to $\mathrm{F}$, while $\mathrm{AR}$ and $\mathrm{S}$ increase. This shows a more significant influence of form on sphericity values than roundness for LBS fragments. Although the AR values of fragment $\mathrm{C}$ and fragment $\mathrm{D}$ are the same, fragment $\mathrm{D}$ is actually more irregular in terms of form. It is because fragment $\mathrm{C}$ has both $\mathrm{EI}$ and $\mathrm{FI}$ equal to 0.42 , while fragment $\mathrm{D}$ has EI and FI equal to 0.44 and 0.40 , respectively. The relatively low AR values of fragments $\mathrm{C}$ and D were caused by two parallel fracture planes generated with small distance. Although fragments $\mathrm{C}$ and D have sharp corners, but they have very small inscribed spheres comparing to its dimension, which results in relative high roundness values comparing with fragments $\mathrm{E}$ and F.

\subsection{Statistical analysis of shape parameters}

It is important to understand the statistical characteristics of particle shape parameters. The cumulative distributions of various shape parameters for LBS particles and LBS fragments are 
shown in Fig. 17. It is found that the shape parameters of LBS fragments have both smaller mean values and wider distributions than those of LBS particles.

Weibull statistics are applied to analyze these shape parameters. The probability of a particle having a shape parameter larger than $L$ is given by $P(L)=\exp \left(-\left(\frac{L}{L_{0}}\right)^{m}\right)$, where $m$ is the Weibull modulus and $L_{0}$ is the characteristic value of the shape parameter. By plotting $\ln (\ln (1 / P))$ against $\ln (L), m$ can be determined from the slope of the best fit line, and $L_{0}$ determined by setting $\ln (\ln (1 / P))=0$. Fig. 18 shows the distributions of all shape parameters and their Weibull fitting lines. It appears that the distributions of the shape parameters could be well fitted by Weibull distribution.

The Weibull modulus, characteristic shape parameters and average shape parameters are summarized in Table. 1. The Weibull modulus rotates the curve, while the scale parameter moves the curve toward left or right. A higher Weibull modulus leads to a steeper distribution function, meaning a lower dispersion of the shape parameters. For LBS particles, all shape parameters have both larger $L_{0}$ and $m$ than LBS fragments. For example, $L_{0}$ reduced from 0.90 to 0.70 and $m$ reduced from 35.29 to 8.48 for the sphericity values after crushing, which indicate a lower average sphericity value and larger dispersion, respectively.

\section{Conclusions}

Recent developments in X-ray $\mu \mathrm{CT}$ have led to the possibility of imaging and characterising particle morphology at a resolution that was unimaginable just a couple of decades ago. In the current context, this advance permits the morphology of particle to be visualised and reconstructed three-dimensionally. This paper proposed a framework to reconstruct particle surface from $\mu \mathrm{CT}$ images by image processing and quantify particle shape by image analysing on reconstructed surface.

The key contribution of this paper is a new 3D roundness evaluation technique that provides detailed insights into the roundness-scale investigation on particle morphology. Four kinds of local curvatures are evaluated from the reconstructed surface composed by triangular meshes. The 3D "corners" were defined as the parts of the particle surface that have large local curvature values than its maximum inscribed sphere. The 3D roundness indexes were then defined through an area-based average approach from all of the particle corners. The key requirement 


\section{Acknowledgements} particle crushing.

is that the reconstructed surface has been properly reconstructed and simplified. It is found that the simplification of reconstructed surface could efficiently eliminate surface texture, and the corners could be well identified by local mean curvature values.

Results have been presented from successful applications of the framework on two kinds of particles: LBS particles and LBS fragments. It is found that the LBS fragments generated from single particle crushing tests have lower mean values and larger dispersion of all the shape parameters than LBS particles. The relationship between sphericity and both form and roundness suggests a different scale influence of form and roundness on particle compactness. In other words, form and roundness have more significant influence on the sphericity values of irregular LBS fragments and regular LBS particles, respectively. The distributions of these shape parameters of both LBS particles and LBS fragments could be well fitted by Weibull statistics. The Weibull modulus and characteristic shape parameter both have significant reduction after

Work is ongoing to improve the $3 \mathrm{D}$ roundness evaluation technique through implementing more sophisticated surface reconstruction and simplification techniques, and defining new roundness indexes. Compared to other descriptors of 3D particle roundness proposed previously, it might be improved through using more surface roundness-scale features by estimating the local curvature. The application of this technique to different granular materials and the investigation of their mechanical behaviour may lead to a better understanding of the influence of particle morphology on mechanical behaviour.

The study presented in this article was supported by General Research Fund No. CityU 120512 from the Research Grant Council of the Hong Kong SAR, Research Grant No. 51379180 from the National Science Foundation of China, and the open-research grant No. SLDRCE15-04 from State Key Laboratory of Civil Engineering Disaster Prenvention of Tongji University. The authors would like to express gratitude to the Advanced Engineering Material Facility (AEMF) of the Hong Kong University of Science and Technology and the Shanghai Yinghua Non-destructive Testing company, who provided the scanning systems of nanome|x system (Phoenix, GE) and v|tome|x m (Phoenix, GE), respectively. 


\section{$1 \quad$ Appendix 1. Abbreviations}

2 AR: representative aspect ratio

$3 \quad$ EI: elongation index

$4 \quad$ FI: flatness index

5 GMC: generalised Marching Cubes

6 LBS: Leighton Buzzard sand

7 MC: Marching Cubes

$8 \quad$ PCA: principal component analysis

$9 \quad$ S: sphericity

10 SA: surface area

$11 \mu \mathrm{CT}$ : micro-computed tomography

12

\section{Appendix 2. Symbols}

$14 a, b, c$ : principal dimensions of a particle $(a \geqslant b \geqslant c)$

$15 A_{n}$ : area of $n^{\text {th }}$ triangular element, which is a part of the corners on a particle

$16 C_{X}$ : convexity

$17 \quad k_{1}, k_{2}$ : principal curvature

$18 \quad k_{G}$ : Gaussian curvature

$19 \quad k_{i}$ : local curvature $\left(k_{1}, k_{2}, k_{M}\right.$ or $\left.k_{G}\right)$ at one vertex

$20 \quad k_{M}$ : mean curvature

$21 \quad k_{S}$ : local curvature $\left(k_{1}, k_{2}, k_{M}\right.$ or $\left.k_{G}\right)$ of the particle's maximum inscribed sphere

$22 L_{0}, m$ : characteristic value and Weibull modulus of the distribution of a shape parameter

$23 \quad R_{1}, R_{2}$ : roundness indexes evaluated from principal curvature

$24 \quad R_{M}$ : roundness index evaluated from mean curvature

$25 \quad R_{G}$ : roundness index evaluated from Gaussian curvature

$26 \quad V$ : volume

$27 \quad V_{C H}$ : volume of convex hull

$28 V_{\text {sphere }}$ : volume of a sphere that has the equivalent surface area to the particle 


\section{$2 \quad$ References}

[1] J. K. Mitchell, K. Soga, Fundamentals of soil behavior, Wiley New York, 1976.

[2] G.-C. Cho, J. Dodds, J. C. Santamarina, Particle shape effects on packing density, stiffness, and strength: natural and crushed sands, Journal of Geotechnical and Geoenvironmental Engineering 132 (5) (2006) 591-602.

[3] P. C. Rousé, R. J. Fannin, D. A. Shuttle, Influence of roundness on the void ratio and strength of uniform sand, Géotechnique 58 (2008) 227-231.

[4] Q. B. Liu, B. M. Lehane, The influence of particle shape on the (centrifuge) cone penetration test (CPT) end resistance in uniformly graded granular soils, Géotechnique 62 (11) (2012) 973-984.

[5] F. N. Altuhafi, M. R. Coop, Changes to particle characteristics associated with the compression of sands, Géotechnique 61 (6) (2010) 459-471.

[6] C. Nouguier-Lehon, B. Cambou, E. Vincens, Influence of particle shape and angularity on the behaviour of granular materials: a numerical analysis, International Journal for Numerical and Analytical Methods in Geomechanics 27 (14) (2003) 1207-1226.

[7] A. A. Pena, R. Garcia-Rojo, H. J. Herrmann, Influence of particle shape on sheared dense granular media, Granular Matter 9 (3-4) (2007) 279-291.

[8] B. Zhou, R. Huang, H. Wang, J. Wang, DEM investigation of particle anti-rotation effects on the micromechanical response of granular materials, Granular Matter 15 (3) (2013) $315-326$.

[9] J. P. de Bono, G. R. McDowell, An insight into the yielding and normal compression of sand with irregularly-shaped particles using DEM, Powder Technology 271 (2015) 270_ 277. 
[10] O. Falagush, G. McDowell, H.-S. Yu, Discrete element modeling of cone penetration tests incorporating particle shape and crushing, International Journal of Geomechanics (2015) 04015003.

[11] F. Altuhafi, C. O’Sullivan, I. Cavarretta, Analysis of an image-based method to quantify the size and shape of sand particles, Journal of Geotechnical and Geoenvironmental Engineering 139 (8) (2013) 1290-1307.

[12] E. List, J. Frenzel, H. Vollstaedt, A new system for single particle strength testing of grinding powders, Industrial diamond review 1 (2006) 42.

[13] S. Kröner, M. T. Doménech Carbó, Determination of minimum pixel resolution for shape analysis: Proposal of a new data validation method for computerized images, Powder Technology 245 (2013) 297-313.

[14] J. Zheng, R. D. Hryciw, Traditional soil particle sphericity, roundness and surface roughness by computational geometry, Géotechnique 65 (6) (2015) 494-506.

[15] E. T. Bowman, K. Soga, W. Drummond, Particle shape characterisation using fourier descriptor analysis, Géotechnique 51 (6) (2001) 545-554.

[16] G. Mollon, J. Zhao, Fourier-voronoi-based generation of realistic samples for discrete modelling of granular materials, Granular Matter 14 (5) (2012) 621-638.

[17] J. Fonseca, C. O’Sullivan, M. R. Coop, P. D. Lee, Non-invasive characterization of particle morphology of natural sands, Soils and Foundations 52 (4) (2012) 712-722.

[18] K. A. Alshibli, A. M. Druckrey, R. I. Al-Raoush, T. Weiskittel, N. V. Lavrik, Quantifying morphology of sands using 3D imaging, Journal of Materials in Civil Engineering.

[19] C. L. Lin, J. D. Miller, 3D characterization and analysis of particle shape using X-ray microtomography (XMT), Powder Technology 154 (1) (2005) 61-69.

[20] J. Lindblad, Surface area estimation of digitized 3d objects using weighted local configurations, Image and Vision Computing 23 (2) (2005) 111-122. 
[21] D. Asahina, M. A. Taylor, Geometry of irregular particles: Direct surface measurements by 3-D laser scanner, Powder Technology 213 (1) (2011) 70-78.

[22] E. J. Garboczi, Three-dimensional mathematical analysis of particle shape using x-ray tomography and spherical harmonics: Application to aggregates used in concrete, Cement and Concrete Research 32 (10) (2002) 1621-1638.

[23] M. A. Taylor, E. J. Garboczi, S. T. Erdogan, D. W. Fowler, Some properties of irregular 3-D particles, Powder Technology 162 (1) (2006) 1-15.

[24] X. Liu, E. J. Garboczi, M. Grigoriu, Y. Lu, S. T. Erdoğan, Spherical harmonic-based random fields based on real particle 3D data: improved numerical algorithm and quantitative comparison to real particles, Powder Technology 207 (1) (2011) 78-86.

[25] E. J. Garboczi, Three dimensional shape analysis of JSC-1A simulated lunar regolith particles, Powder Technology 207 (1) (2011) 96-103.

[26] J. W. Bullard, E. J. Garboczi, Defining shape measures for 3D star-shaped particles: Sphericity, roundness, and dimensions, Powder Technology 249 (2013) 241-252.

[27] B. Zhou, J. Wang, B. Zhao, Micromorphology characterization and reconstruction of sand particles using micro x-ray tomography and spherical harmonics, Engineering Geology 184 (14) (2014) 126-137.

[28] S. R. Stock, Recent advances in X-ray microtomography applied to materials, International Materials Reviews 53 (3) (2008) 129-181.

[29] B. Zhao, J. Wang, M. Coop, G. Viggiani, M. Jiang, An investigation of single sand particle fracture using X-ray micro-tomography, Géotechnique 65 (8) (2015) 625-641.

[30] R. C. Gonzalez, R. E. Woods, Digital image processing, Upper Saddle River, N.J.: Pearson/Prentice Hall, 2010.

[31] N. Otsu, A threshold selection method from gray-level histograms, IEEE Trans. Systems, Man and Cybernetics 9 (1) (1979) 62-66. 
[32] W. E. Lorensen, H. E. Cline, Marching cubes: A high resolution 3D surface construction algorithm, in: Computer graphics, Vol. 21, 1987, pp. 163-169.

[33] W. Heiden, T. Goetze, J. Brickmann, Fast generation of molecular surfaces from 3D data fields with an enhanced "marching cube" algorithm, Journal of Computational Chemistry 14 (2) (1993) 246-250.

[34] P. J. Yim, G. B. C. Vasbinder, V. B. Ho, P. L. Choyke, Isosurfaces as deformable models for magnetic resonance angiography, Medical Imaging, IEEE Transactions on 22 (7) (2003) 875-881.

[35] F. Lin, H. S. Seah, Y. T. Lee, Deformable volumetric model and isosurface: exploring a new approach for surface boundary construction, Computers \& graphics 20 (1) (1996) $33-40$.

[36] D. A. Field, Laplacian smoothing and delaunay triangulations, Communications in applied numerical methods 4 (6) (1988) 709-712.

[37] L. P. Kobbelt, M. Botsch, U. Schwanecke, H.-P. Seidel, Feature sensitive surface extraction from volume data, in: Proceedings of the 28th annual conference on Computer graphics and interactive techniques, 2001, pp. 57-66.

[38] H.-C. Hege, D. Stalling, M. Seebass, M. Zockler, A generalized marching cubes algorithm based on non-binary classifications, in: Preprint SC 97-05, Konrad-Zuse-Zentrum für Informationstechnik Berlin, Germany, 1997.

[39] D. Stalling, M. Zoeckler, H.-C. Hege, Interactive segmentation of 3D medical images with subvoxel accuracy, in: Proc. CAR'98 Computer Assisted Radiology and Surgery, 1998.

[40] P. J. Barrett, The shape of rock particles, a critical review, Sedimentology 27 (3) (1980) 291-303.

[41] I. Cavarretta, M. R. Coop, C. O'Sullivan, The influence of particle characteristics on the behaviour of coarse grained soils, Géotechnique 60 (6) (2010) 413-423. 
[42] K. Senetakis, M. R. Coop, M. C. Todisco, Tangential load-deflection behaviour at the contacts of soil particles, Géotechnique Letters 3 (April-June) (2013) 59-66.

[43] S. J. Blott, K. Pye, Particle shape: a review and new methods of characterization and classification, Sedimentology 55 (1) (2008) 31-63.

[44] T. Zingg, Beitrag zur schotteranalyse, Schweizerische Mineralogische und Petrographische Mitteilungen 15 (1935) 52-56.

[45] A. B. Phillion, P. D. Lee, E. Maire, S. L. Cockcroft, Quantitative assessment of deformation-induced damage in a semisolid aluminum alloy via X-ray microtomography, Metallurgical and Materials Transactions A 39 (10) (2008) 2459-2469.

[46] Y. Sun, B. Indraratna, S. Nimbalkar, Three-dimensional characterisation of particle size and shape for ballast, Géotechnique Letters 4 (2014) 197-202.

[47] B. Sukumaran, A. Ashmawy, Quantitative characterisation of the geometry of discret particles, Géotechnique 51 (7) (2001) 619-627.

[48] P. Lindstrom, G. Turk, Fast and memory efficient polygonal simplification, in: Visualization'98. Proceedings, IEEE, 1998, pp. 279-286.

[49] M. P. Do Carmo, Differential geometry of curves and surfaces, Vol. 2, Prentice-Hall Englewood Cliffs, 1976.

[50] X. Chen, F. Schmitt, Intrinsic surface properties from surface triangulation, in: Computer VisionECCV'92, 1992, pp. 739-743.

[51] C. Dong, G. Wang, Curvatures estimation on triangular mesh, Journal of Zhejiang University Scinence 6 (1) (2005) 128-136.

[52] H. Wadell, Volume, shape, and roundness of rock particles, The Journal of Geology 40 (1932) 443-451.

[53] C. R. I. Clayton, C. O. R. Abbireddy, R. Schiebel, A method of estimating the form of coarse particulates, Géotechnique 59 (6) (2009) 493-501. 


\section{$1 \quad$ List of Figures}

1 Flowchart illustrating image processing and analysing with X-ray $\mu \mathrm{CT}$ images

2 Illustration of image processing: (a) close-up raw gray image; (b) close-up image after the median filter; (c) close-up image after thresholding segmentation; (d) image of LBS particles after thresholding segmentation.

3 Illustration of surface reconstruction: (a-c) LBS particle morphology represented by voxel assembly, reconstructed surface generated from MC, and smooth reconstructed surface generated from GMC with a kernel size of 5 voxels; (d-f) close-up views.

4 Three scales of particle shape (following Barrett[1980]).

5 (a) Local surface geometry around point $p$; (b) one-ring and two-ring neighborhood of point $p$.

6 Illustration of finding maximum inscribed sphere: (a-b) distance maps of LBS particle and LBS fragment; (b-d) 3D view of LBS particle and LBS fragment with their maximum inscribed spheres.

7 Corners identified by different curvatures on a LBS particle surface: (a) maximum principal curvature $\left(k_{1}\right)\left(\mathrm{mm}^{-1}\right)$; (b) minimum principal curvature $\left(k_{2}\right)$ $\left(\mathrm{mm}^{-1}\right)$; (c) mean curvature $\left(k_{M}\right)\left(\mathrm{mm}^{-1}\right)$; (d) Gaussian curvature $\left(k_{G}\right)\left(\mathrm{mm}^{-2}\right)$.

8 Reconstructed surfaces with different numbers of faces for corner identification by mean curvature: (a-d) LBS particle with 10,000, 4,000, 1,500 and 500 faces (corners have mean curvature larger than or equal to $1.44 \mathrm{~mm}^{-1}$ ); (e-h) LBS fragment with 10,000, 4,000, 1,500 and 500 faces (corners have mean curvature larger than or equal to $4.15 \mathrm{~mm}^{-1}$ ).

9 Distributions of mean curvature for reconstructed surfaces with different numbers of faces for (a) LBS particle and (b) LBS fragment.

10 Three-dimensional view of reconstructed surfaces with different sphericity values: (a-d) LBS particles, $\mathrm{S}=0.94,0.90,0.85$ and 0.80; (e-h) LBS fragments, $\mathrm{S}$ $=0.82,0.72,0.60$ and 0.50 .

11 Aspect ratios of measured particles (EI: elongation index; FI: flatness index). 
12 Relationship between $R_{G}$ and other roundness values for (a) LBS particles; (b) LBS fragments.

13 Relationship between volume and surface area of (a) LBS particles and (b) LBS fragments.

5

14 Relationship between sphericity and convexity.

15 Contour plot of sphericity from AR and $R_{M}$.

16 Three-dimensional view of reconstructed surfaces showing mean curvature values $\left(\mathrm{mm}^{-1}\right)$ at corners along with their shape parameters.

17 Cumulative distributions of shape parameters for (a) LBS particles and (b) LBS fragments.

18 Cumulative distributions of shape parameters for LBS particles and LBS fragments on Weibull plots with fitting lines. 


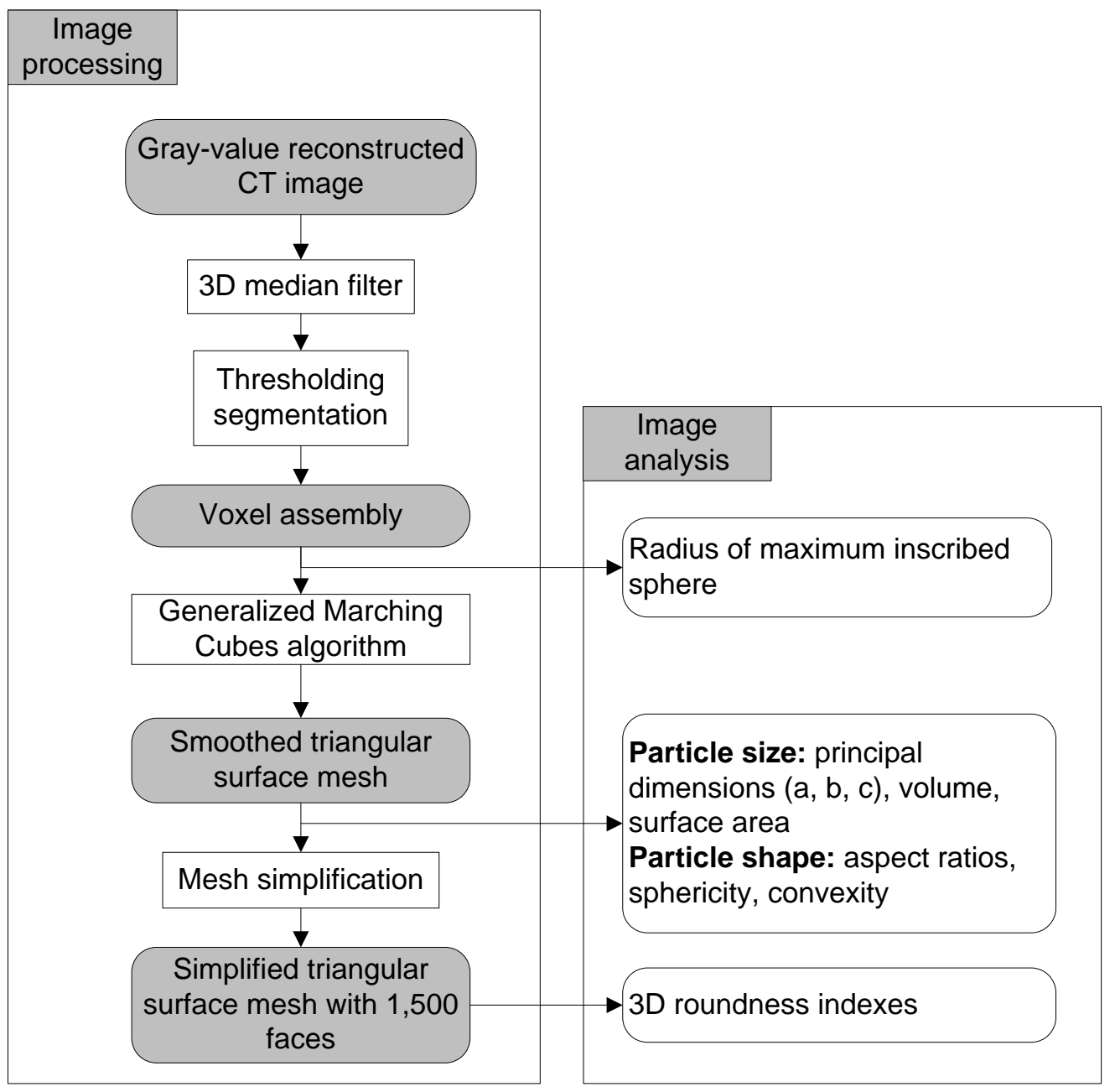

Figure 1: Flowchart illustrating image processing and analysing with $\mathrm{X}$-ray $\mu \mathrm{CT}$ images 


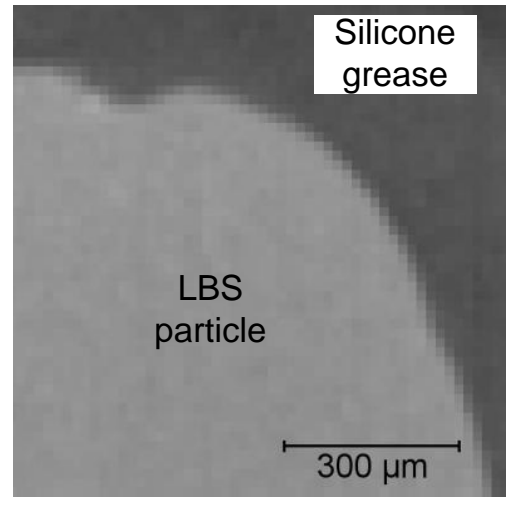

(a)

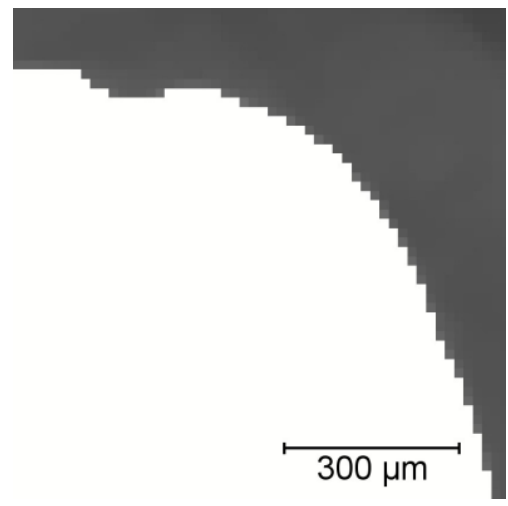

(c)

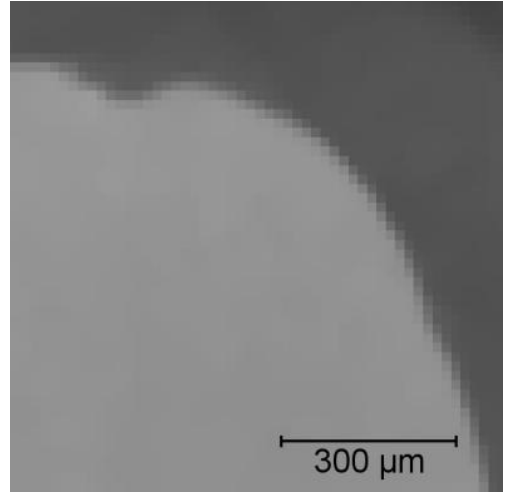

(b)

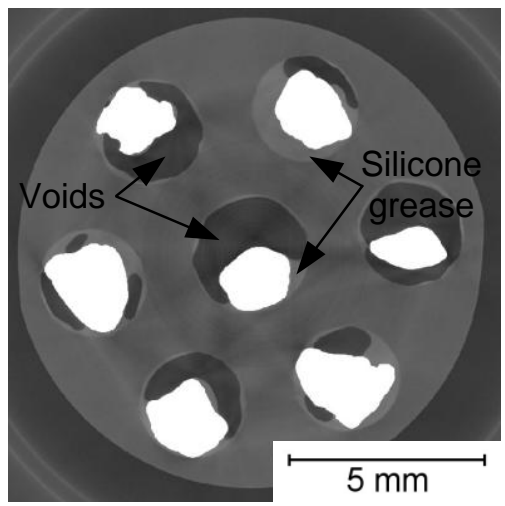

(d)

Figure 2: Illustration of image processing: (a) close-up raw gray image; (b) close-up image after the median filter; (c) close-up image after thresholding segmentation; (d) image of LBS particles after thresholding segmentation. 


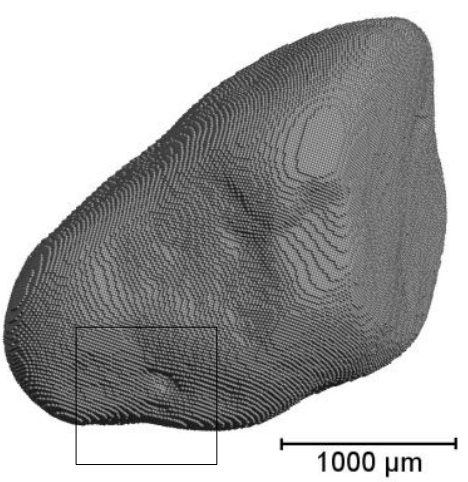

(a)

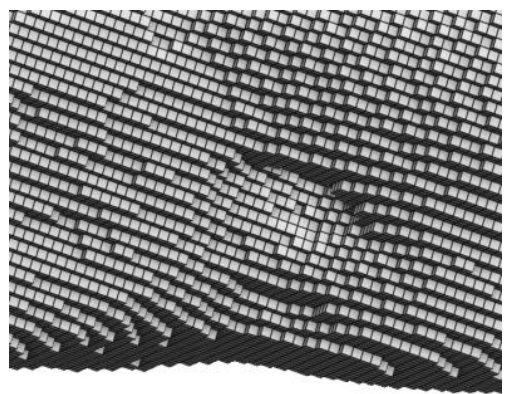

$200 \mu \mathrm{m}$

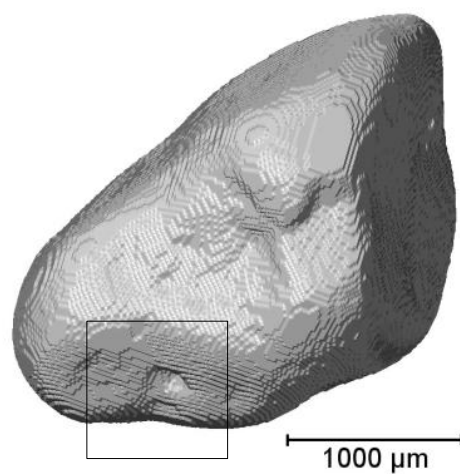

(b)

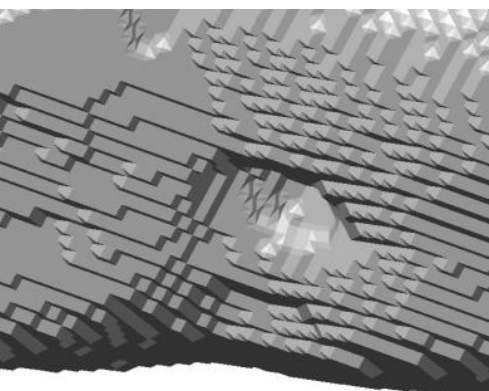

$200 \mu \mathrm{m}$

(e)

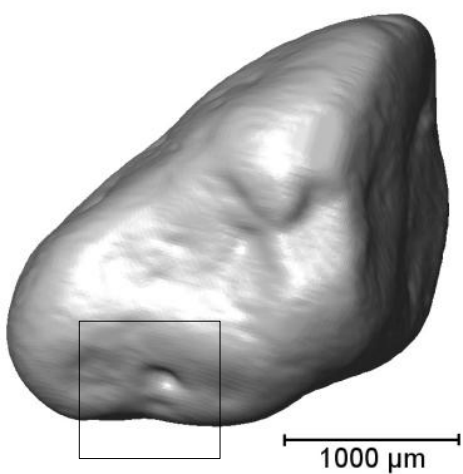

(c)

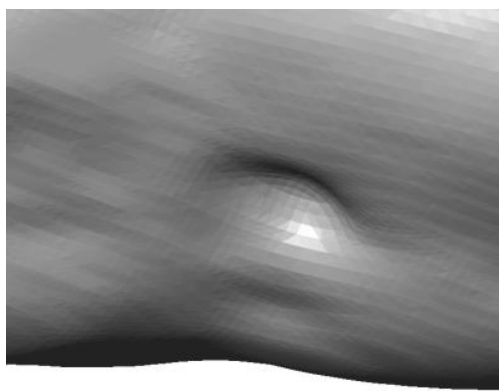

$200 \mu \mathrm{m}$

(f)

Figure 3: Illustration of surface reconstruction: (a-c) LBS particle morphology represented by voxel assembly, reconstructed surface generated from MC, and smooth reconstructed surface generated from GMC with a kernel size of 5 voxels; (d-f) close-up views. 


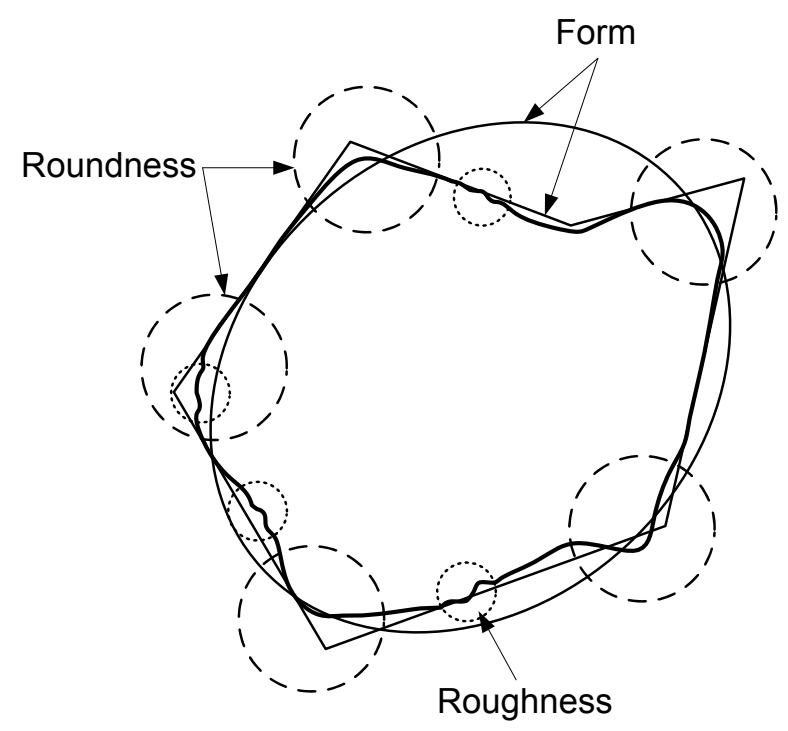

Figure 4: Three scales of particle shape (following Barrett[1980]). 

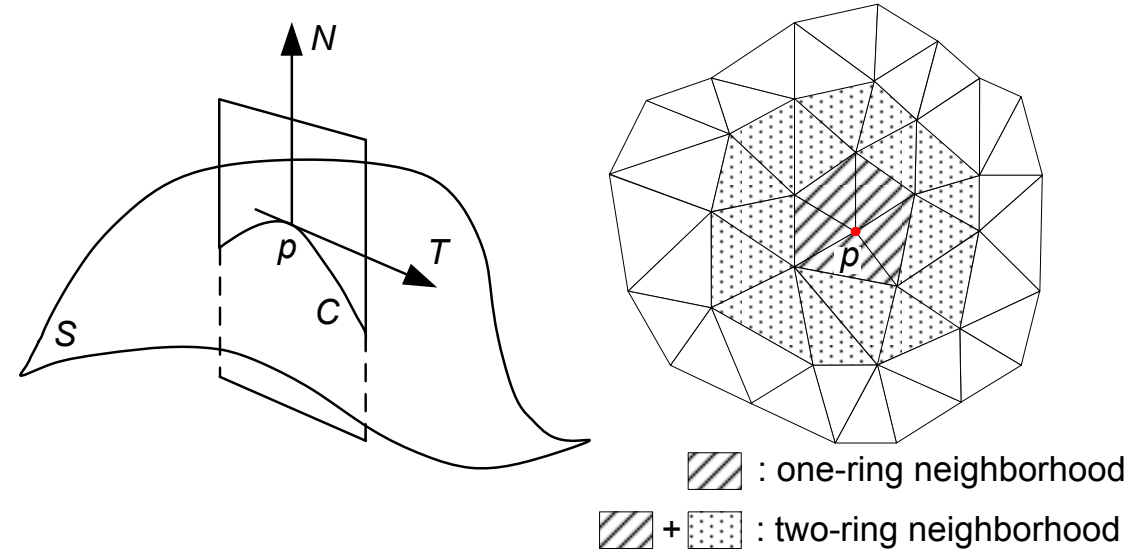

(a)

(b)

Figure 5: (a) Local surface geometry around point $p$; (b) one-ring and two-ring neighborhood of point $p$. 


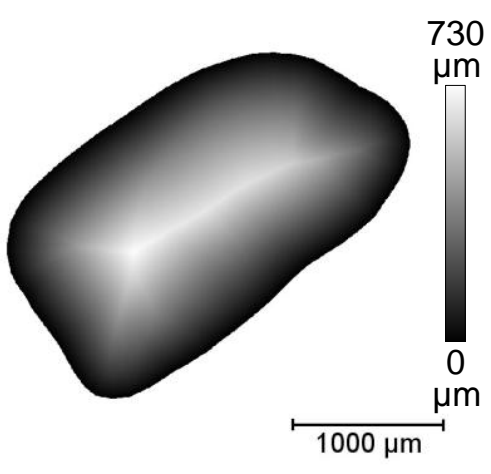

(a)

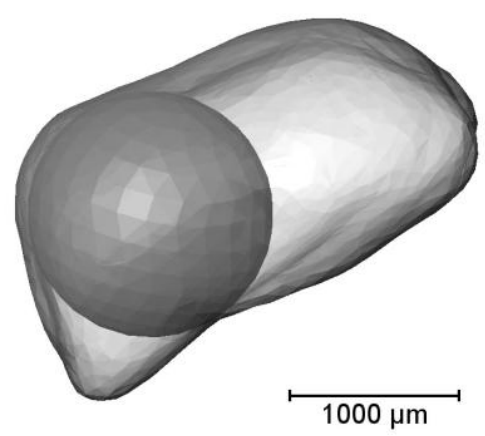

(c)

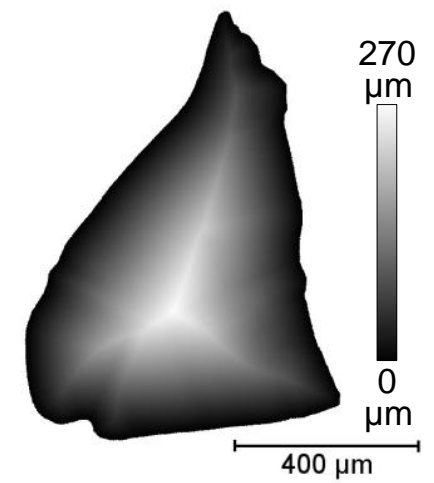

(b)

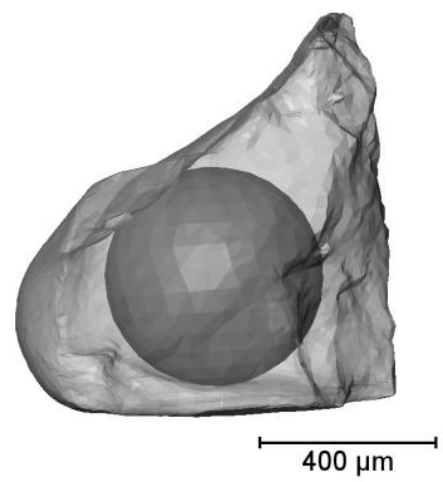

(d)

Figure 6: Illustration of finding maximum inscribed sphere: (a-b) distance maps of LBS particle and LBS fragment; (b-d) 3D view of LBS particle and LBS fragment with their maximum inscribed spheres. 


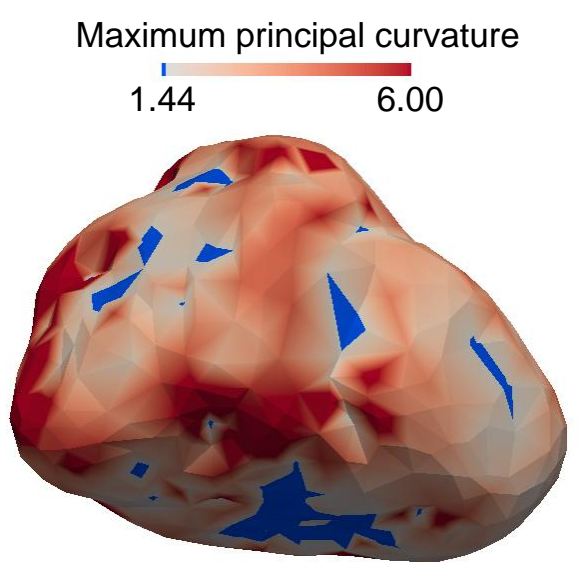

(a)

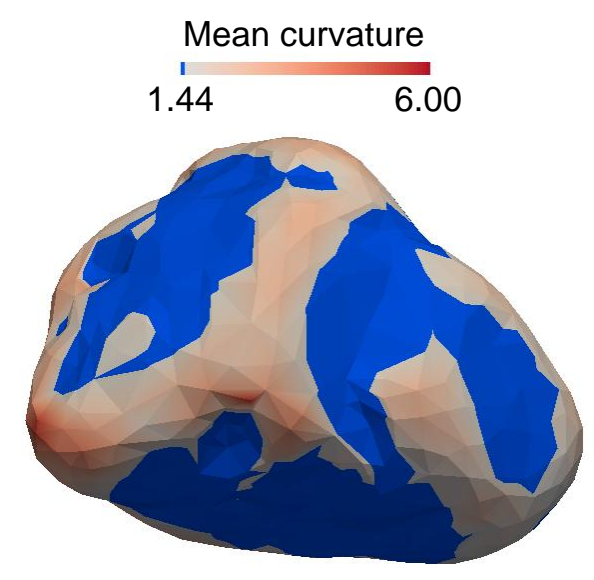

(c)

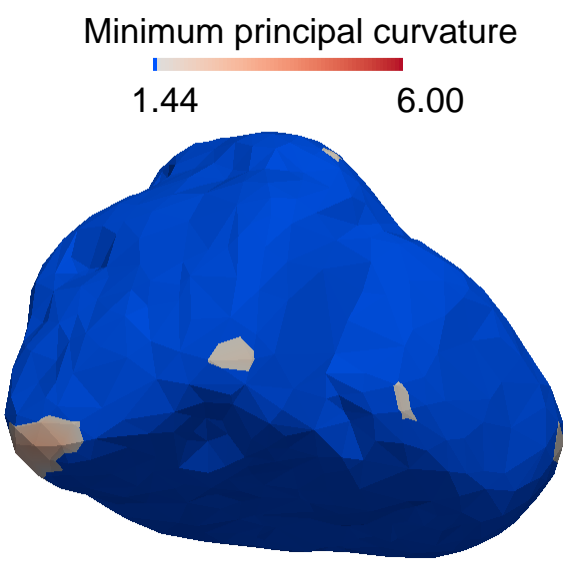

(b)

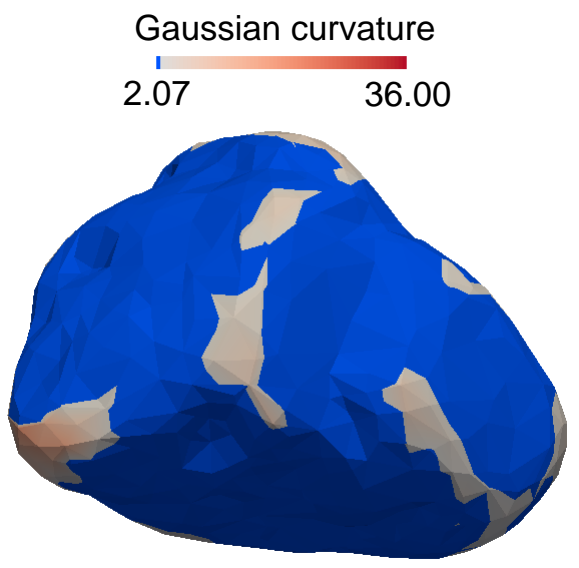

(d)

Figure 7: Corners identified by different curvatures on a LBS particle surface: (a) maximum principal curvature $\left(k_{1}\right)\left(\mathrm{mm}^{-1}\right)$; (b) minimum principal curvature $\left(k_{2}\right)\left(\mathrm{mm}^{-1}\right)$; (c) mean curvature $\left(k_{M}\right)\left(\mathrm{mm}^{-1}\right) ;(\mathrm{d})$ Gaussian curvature $\left(k_{G}\right)\left(\mathrm{mm}^{-2}\right)$. 


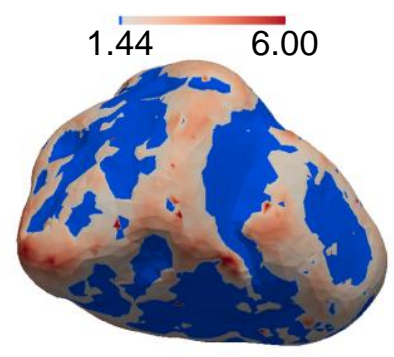

(a)

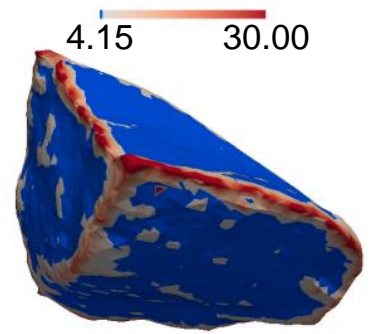

(e)

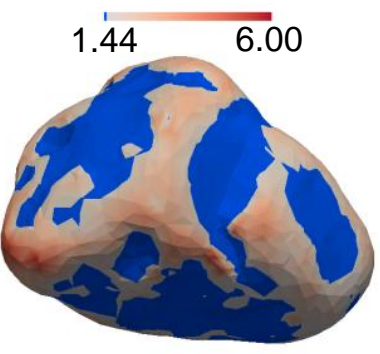

(b)

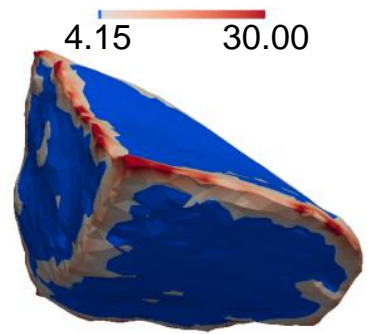

(f)

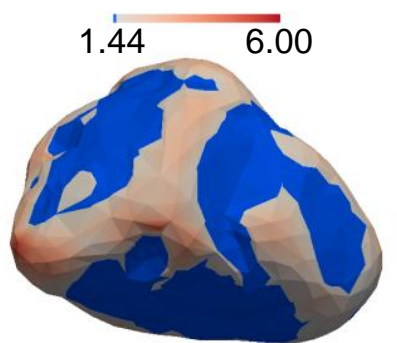

(c)

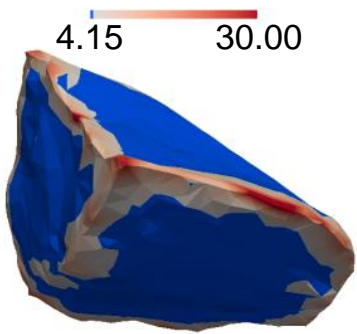

(g)

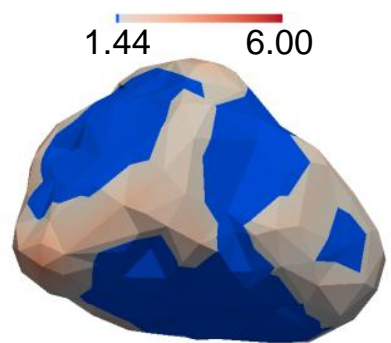

(d)

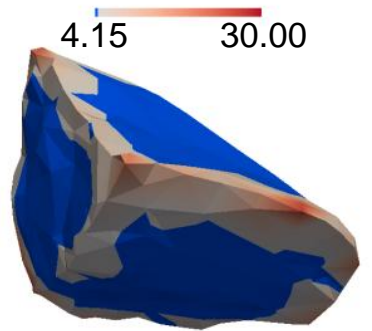

(h)

Figure 8: Reconstructed surfaces with different numbers of faces for corner identification by mean curvature: (a-d) LBS particle with 10,000, 4,000, 1,500 and 500 faces (corners have mean curvature larger than or equal to $1.44 \mathrm{~mm}^{-1}$ ); (e-h) LBS fragment with 10,000, 4,000, 1,500 and 500 faces (corners have mean curvature larger than or equal to $4.15 \mathrm{~mm}^{-1}$ ). 


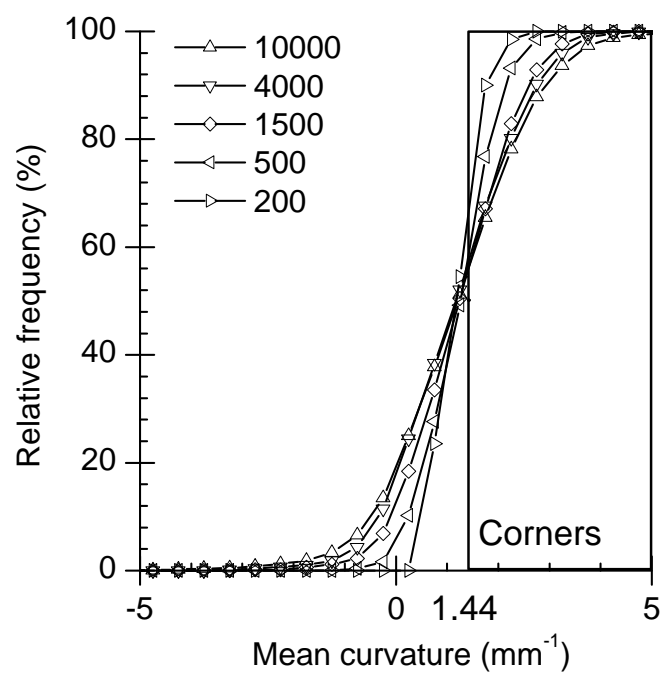

(a)

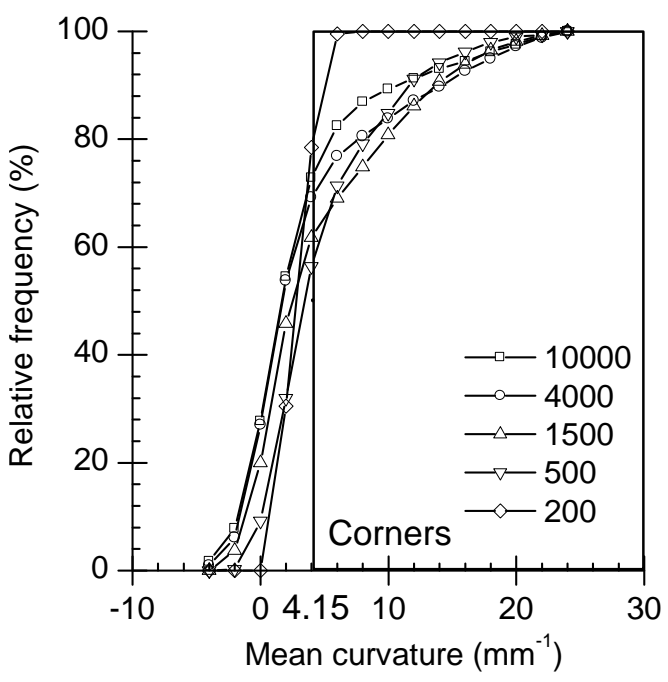

(b)

Figure 9: Distributions of mean curvature for reconstructed surfaces with different numbers of faces for (a) LBS particle and (b) LBS fragment. 


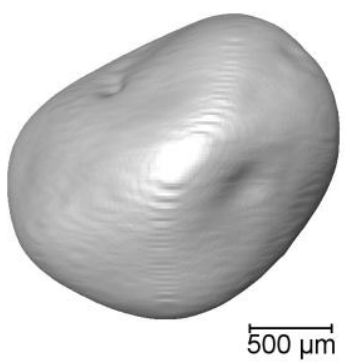

(a)

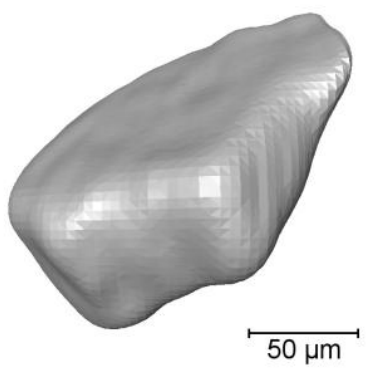

(e)

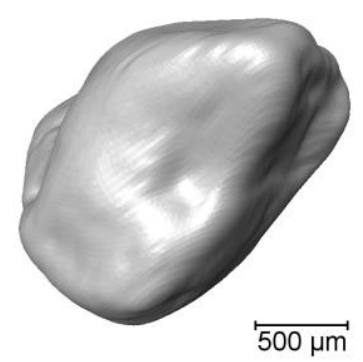

(b)

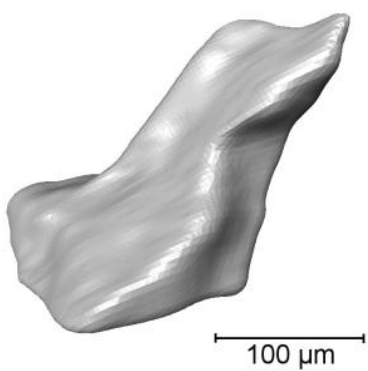

(f)

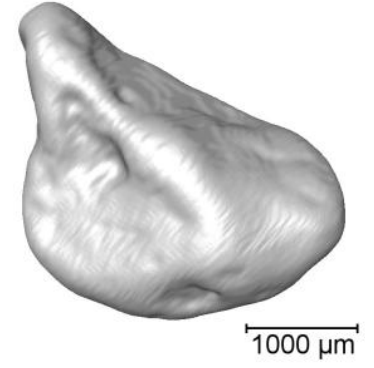

(c)

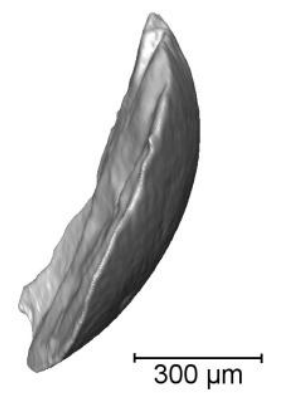

(g)

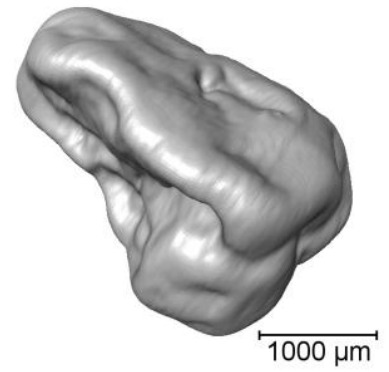

(d)

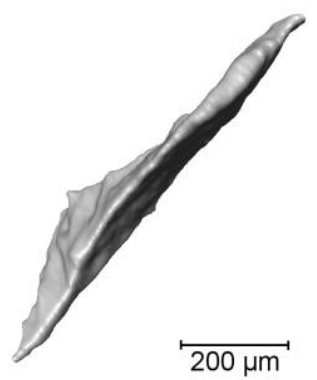

(h)

Figure 10: Three-dimensional view of reconstructed surfaces with different sphericity values: (a-d) LBS particles, $\mathrm{S}=0.94,0.90,0.85$ and 0.80; (e-h) LBS fragments, $\mathrm{S}=0.82,0.72,0.60$ and 0.50 . 


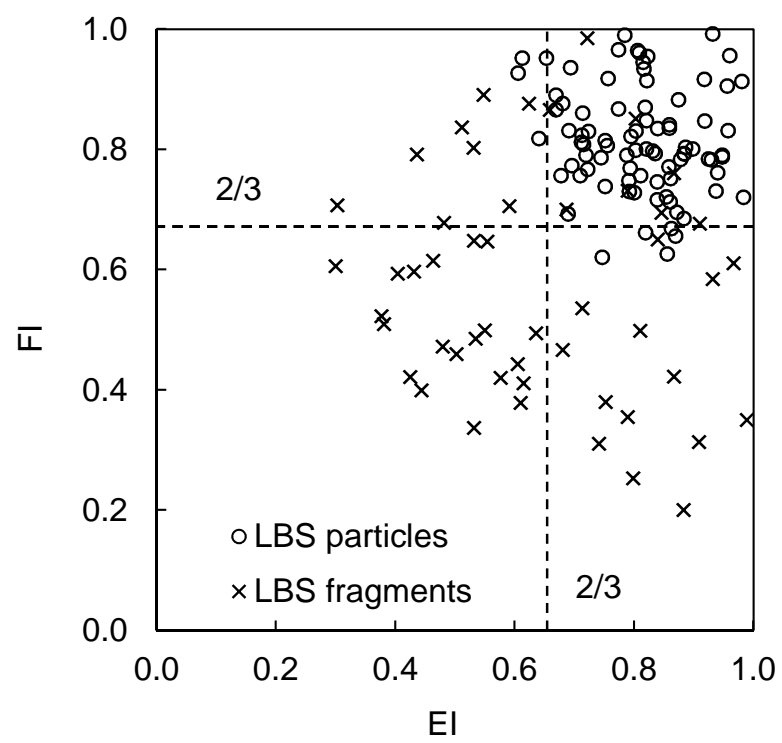

Figure 11: Aspect ratios of measured particles (EI: elongation index; FI: flatness index). 


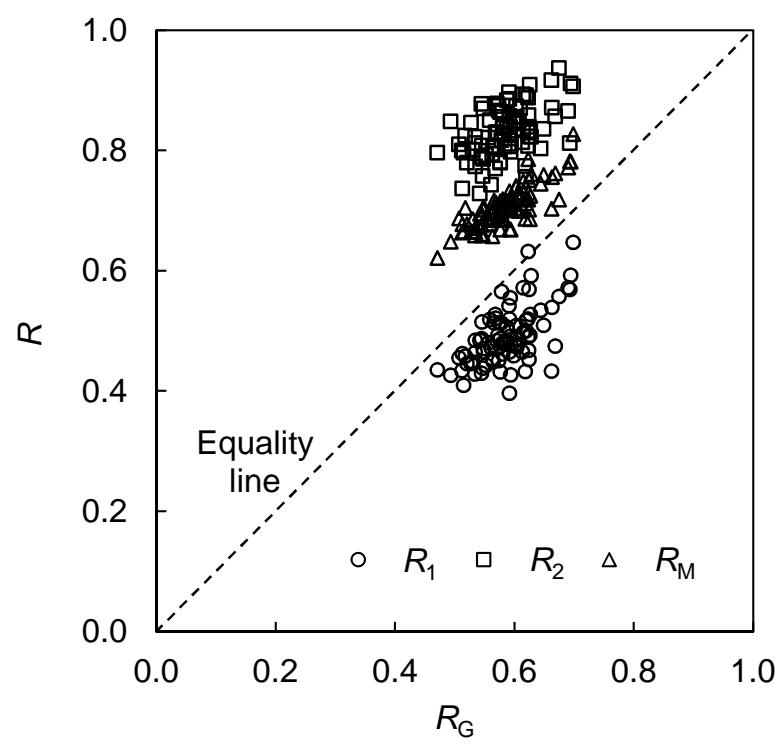

(a)

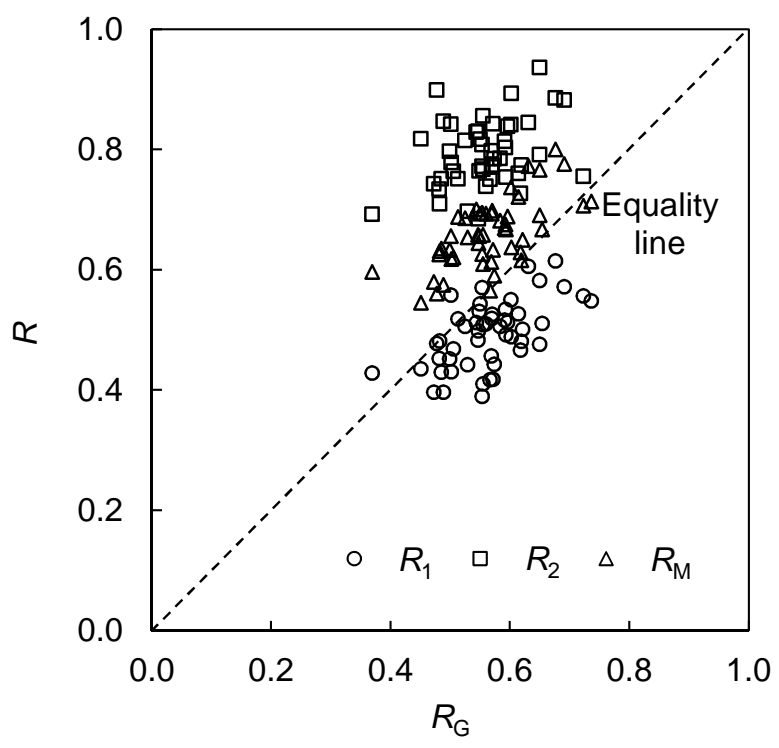

(b)

Figure 12: Relationship between $R_{G}$ and other roundness values for (a) LBS particles; (b) LBS fragments. 


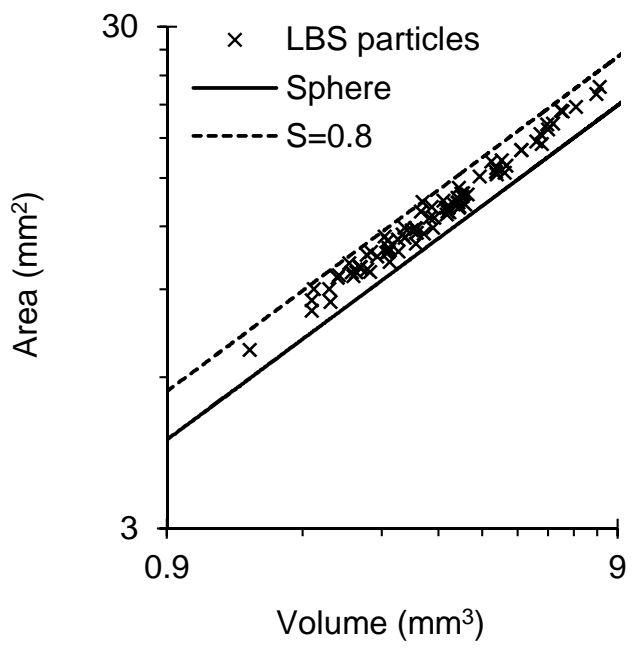

(a)

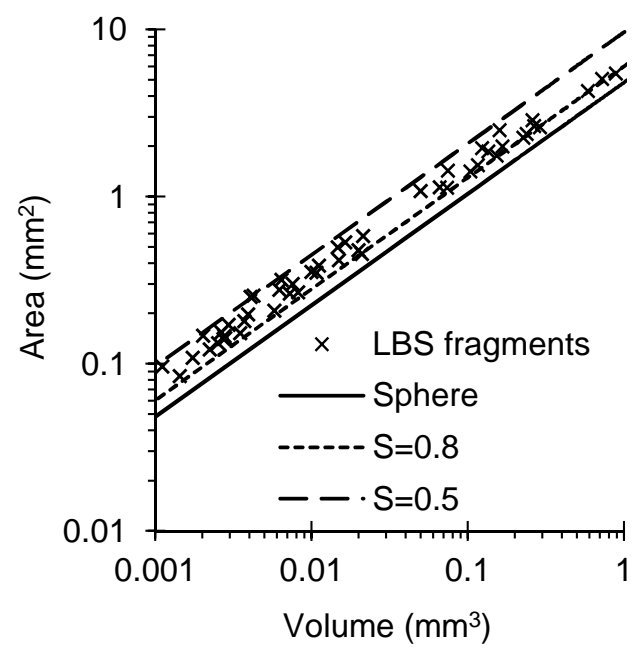

(b)

Figure 13: Relationship between volume and surface area of (a) LBS particles and (b) LBS fragments. 


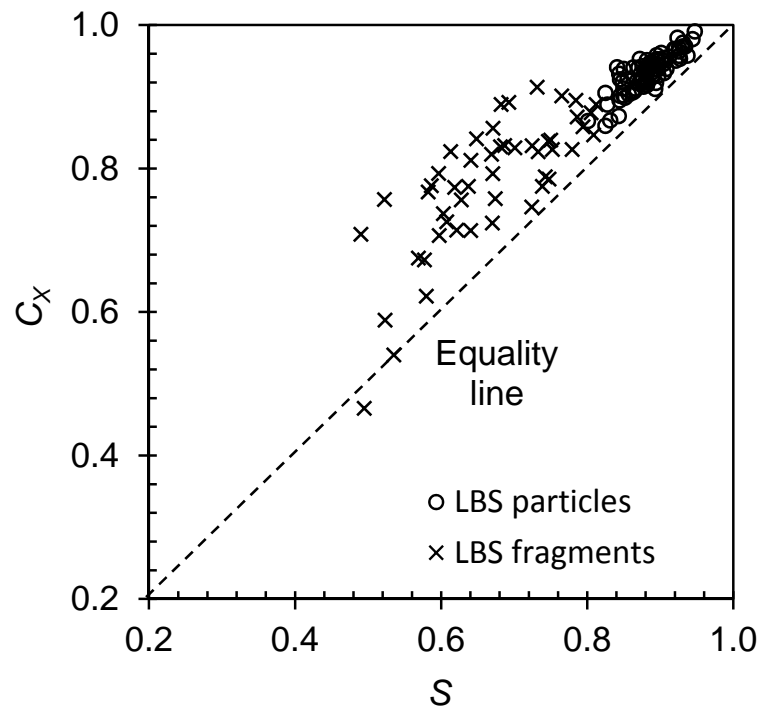

(a)

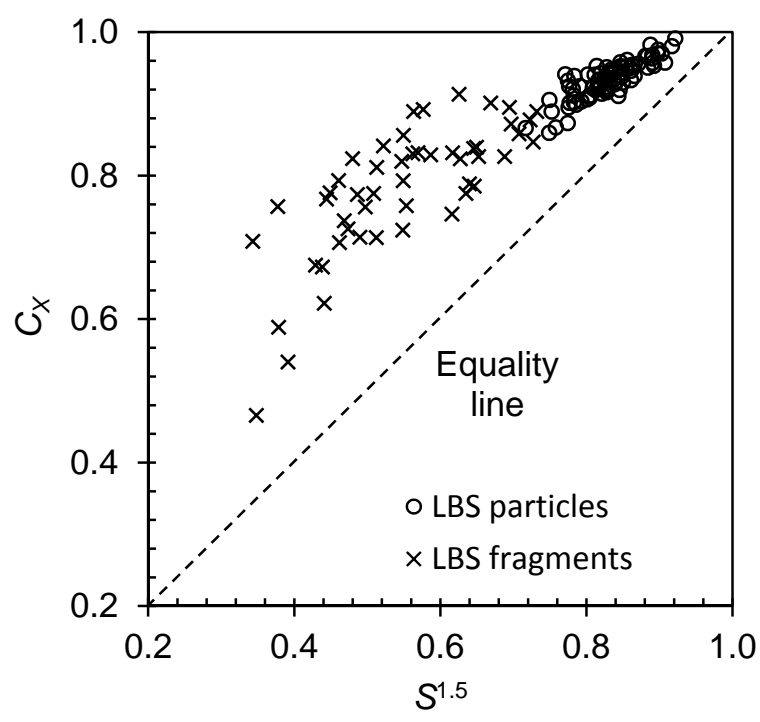

(b)

Figure 14: Relationship between sphericity and convexity. 


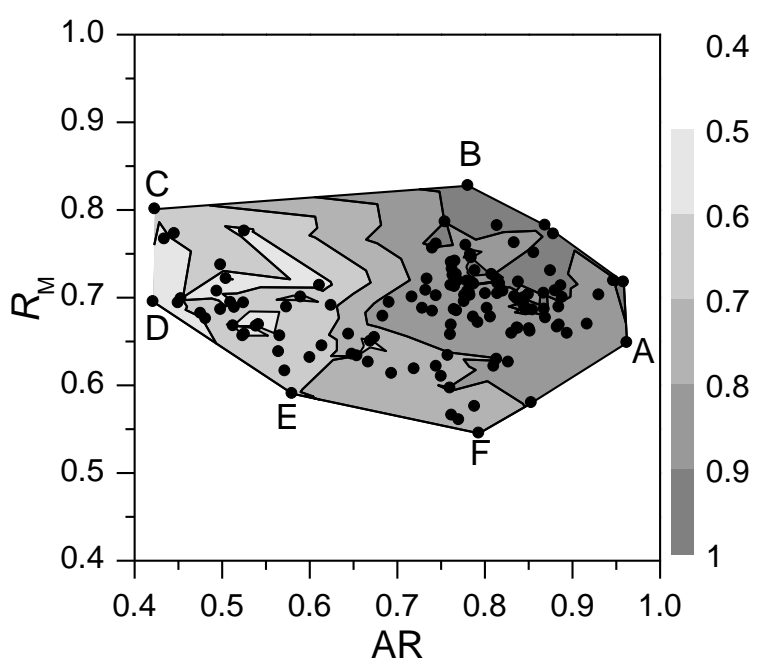

Figure 15: Contour plot of sphericity from $\mathrm{AR}$ and $R_{M}$. 
A

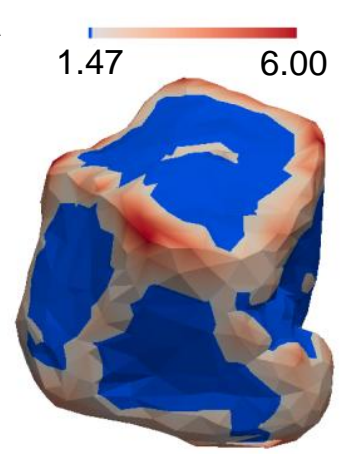

AR: $0.96 ; R_{M}: 0.65$

S: 0.88 ; CX: 0.92

D

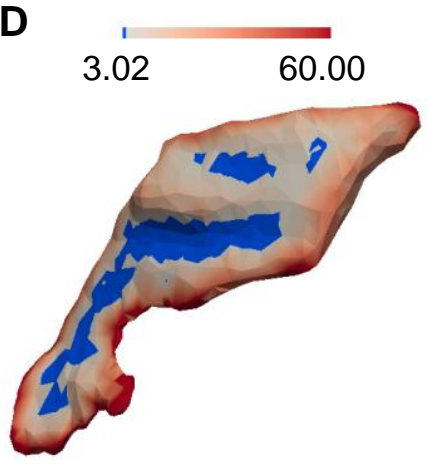

AR: $0.42 ; R_{M}: 0.70$

S: 0.52 ; CX: 0.59
B

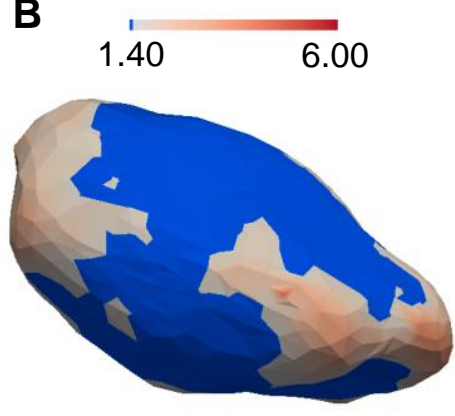

AR: $0.78 ; R_{M}: 0.85$

S: 0.95 ; CX: 0.99

E

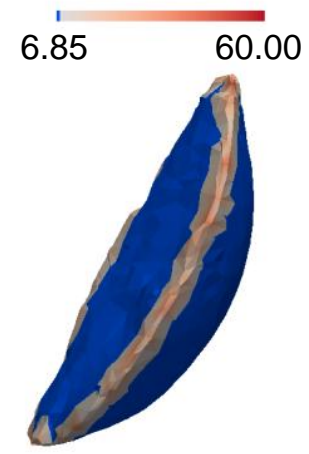

AR: $0.58 ; R_{M}: 0.59$

S: 0.69 ; CX: 0.89
C

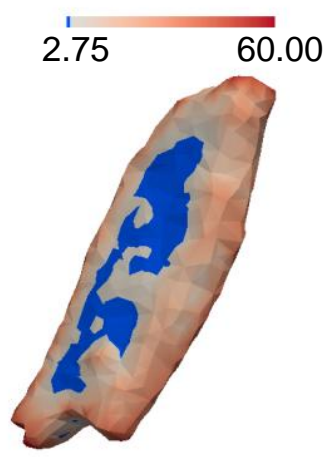

AR: $0.42 ; R_{M}: 0.80$

S: 0.65 ; CX: 0.84

$\mathbf{F}$
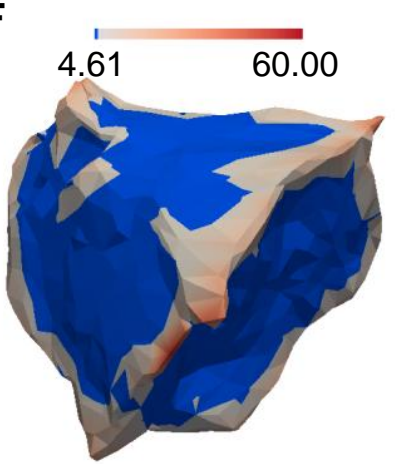

AR: $0.79 ; R_{M}: 0.54$

S: 0.74 ; CX: 0.79

Figure 16: Three-dimensional view of reconstructed surfaces showing mean curvature values $\left(\mathrm{mm}^{-1}\right)$ at corners along with their shape parameters. 


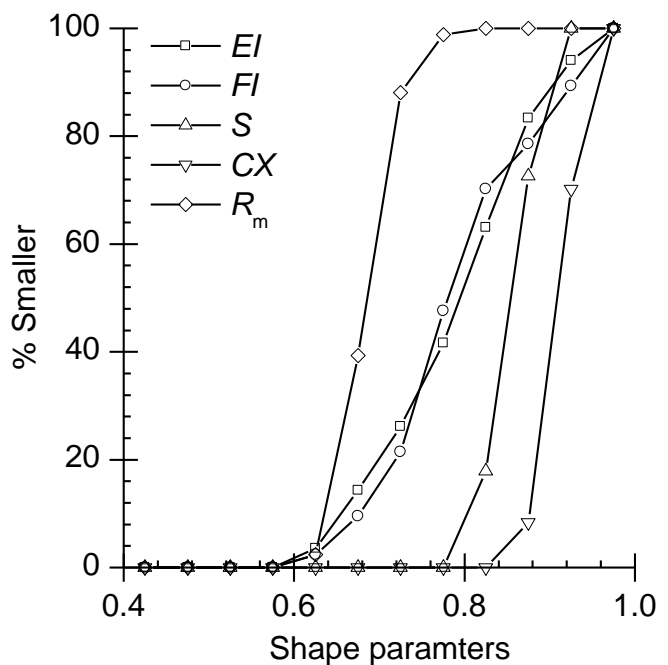

(a)

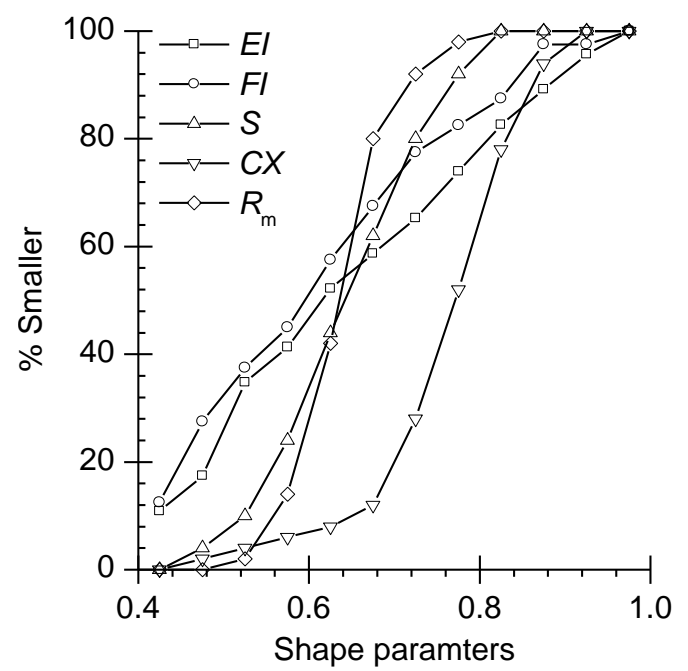

(b)

Figure 17: Cumulative distributions of shape parameters for (a) LBS particles and (b) LBS fragments. 


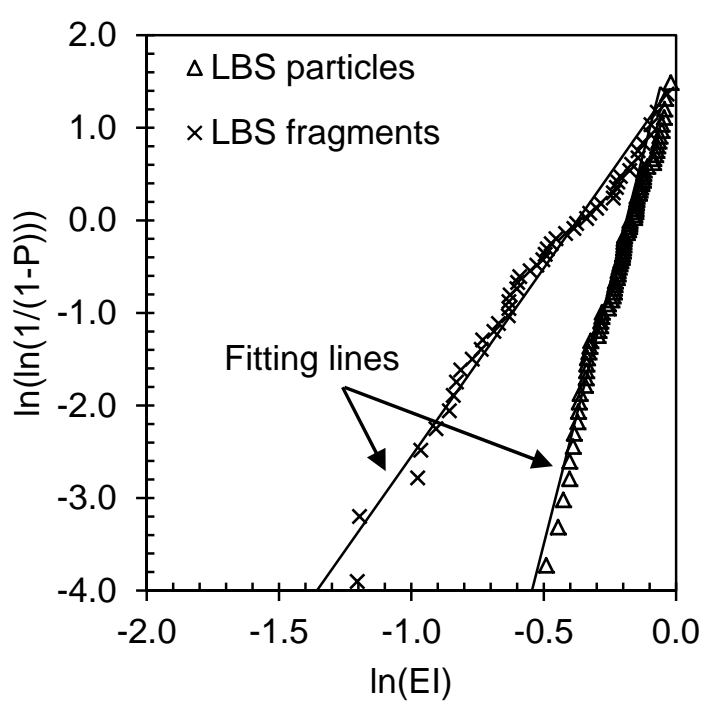

(a)

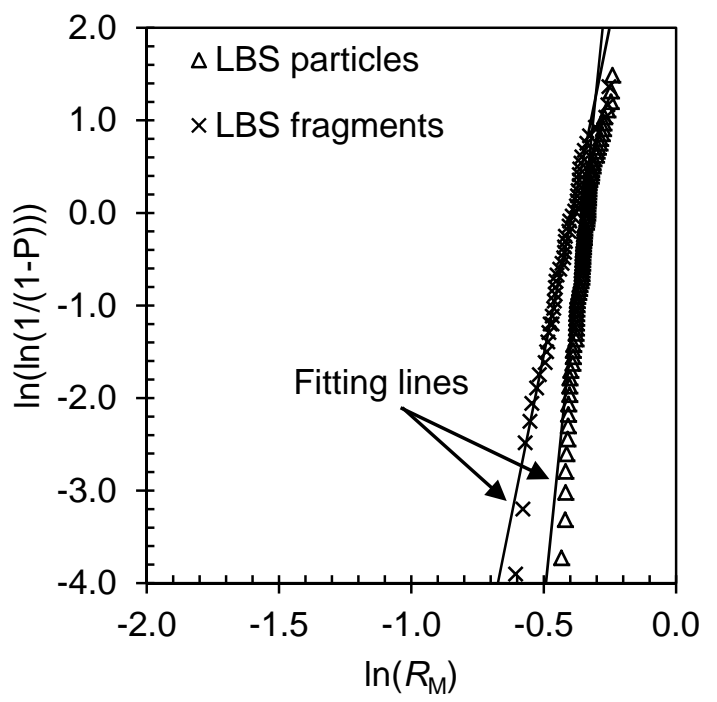

(c)

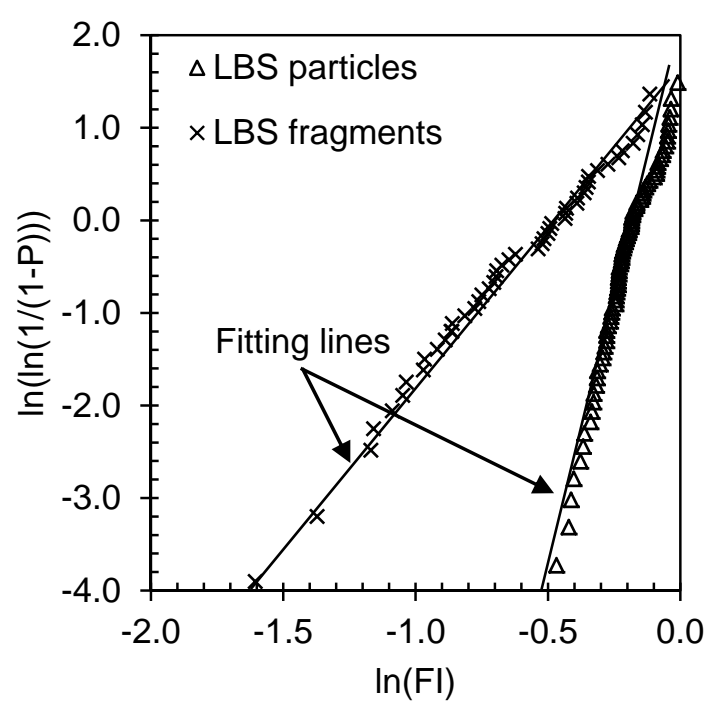

(b)

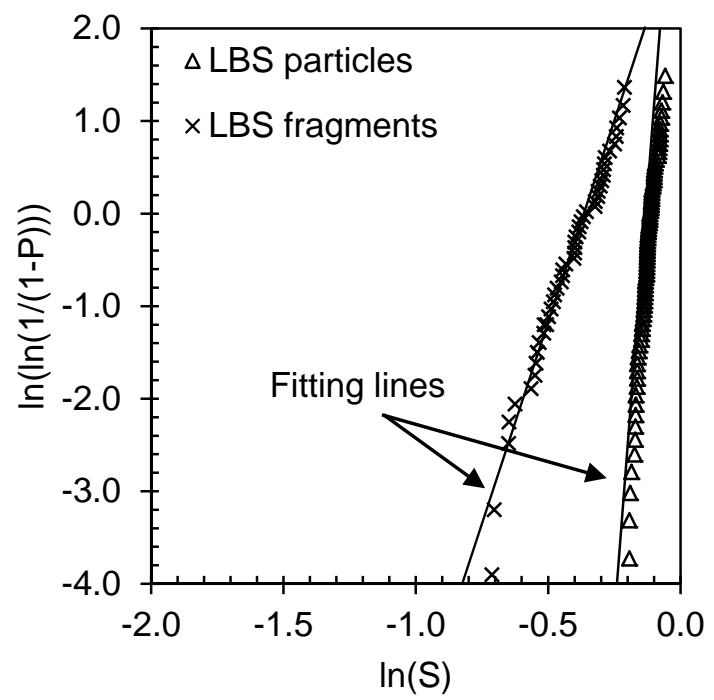

(d)

Figure 18: Cumulative distributions of shape parameters for LBS particles and LBS fragments on Weibull plots with fitting lines. 
Table 1: Weibull modulus, characteristic shape parameters and average shape parameters.

\begin{tabular}{cccccc}
\hline Sand & Parameters & $m$ & $L_{0}$ & $\bar{L}$ & $R^{2}$ \\
\hline \multirow{4}{*}{ LBS particles } & EI & 10.49 & 0.85 & 0.81 & 0.98 \\
& FI & 11.05 & 0.85 & 0.82 & 0.96 \\
& $R_{M}$ & 25.34 & 0.72 & 0.71 & 0.92 \\
& $\mathrm{~S}$ & 35.29 & 0.90 & 0.88 & 0.97 \\
\hline \multirow{5}{*}{ LBS fragments } & EI & 3.88 & 0.70 & 0.64 & 0.98 \\
& FI & 3.28 & 0.62 & 0.57 & 0.99 \\
& $R_{M}$ & 13.67 & 0.68 & 0.66 & 0.96 \\
& $\mathrm{~S}$ & 8.48 & 0.70 & 0.67 & 0.98 \\
\hline
\end{tabular}




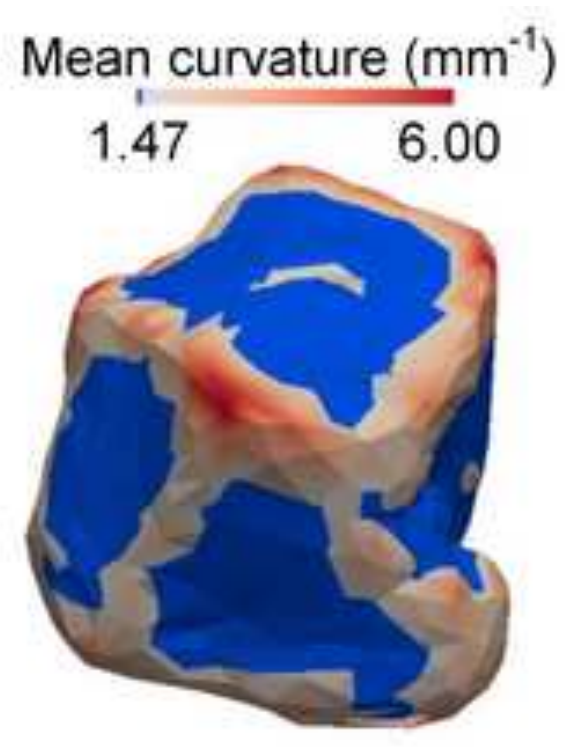

AR: $0.96 ; R_{M}: 0.65$ S: 0.88 ; CX: 0.92

Mean curvature $\left(\mathrm{mm}^{-1}\right)$ 3.02 60.00

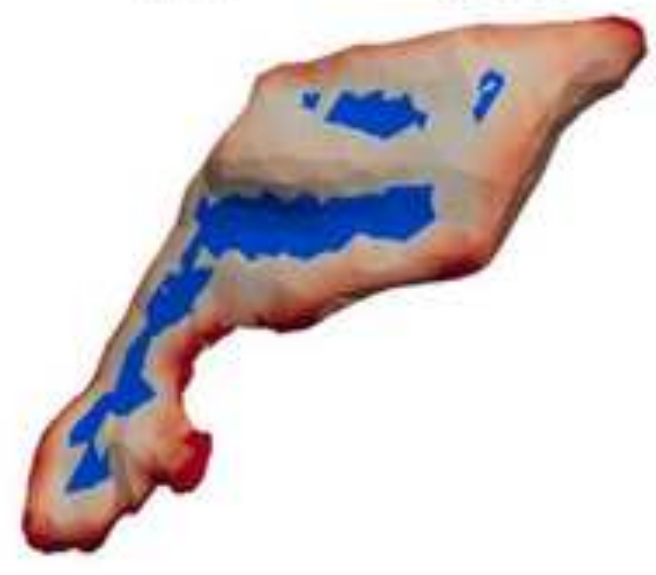

AR: $0.42 ; R_{M}: 0.70$ S: $0.52 ; C X: 0.59$
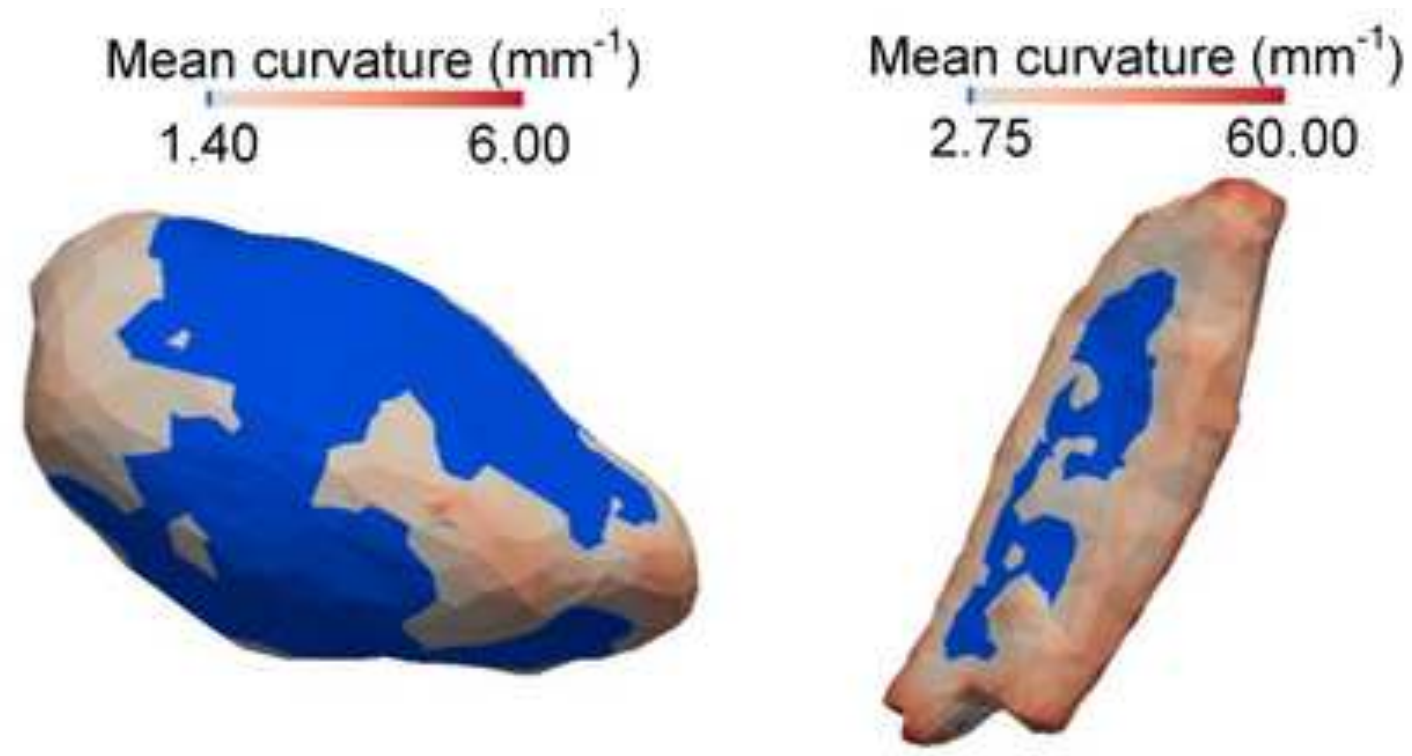

AR: $0.78 ; R_{M}: 0.85$ S: $0.95 ; C X: 0.99$

Mean curvature $\left(\mathrm{mm}^{-1}\right)$

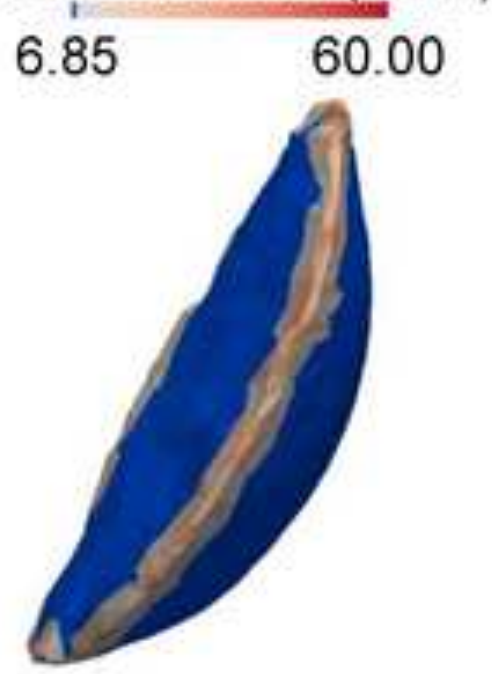

AR: $0.58 ; R_{M}: 0.59$ S: $0.69 ; C X: 0.89$
AR: $0.42 ; R_{M}: 0.80$ S: $0.65 ; \mathrm{CX}: 0.84$ Mean curvature $\left(\mathrm{mm}^{-1}\right)$

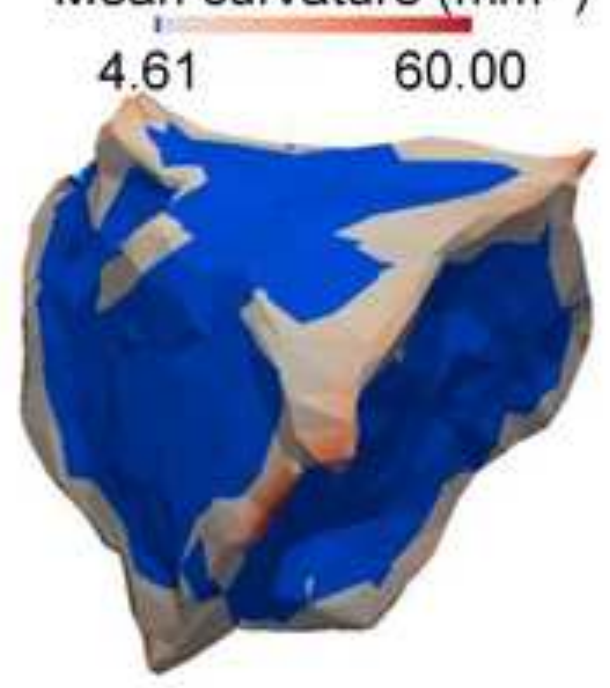

AR: $0.79 ; R_{M}: 0.54$ S: $0.74 ; C X: 0.79$ 